\title{
Decoherence of a Pointer by a Gas Reservoir
}

\author{
Michael Kleckner* and Amiram Ron \\ Department of Physics, \\ Technion - Israel Institute of Technology, \\ Haifa 32000, Israel
}

(November 19, 2018)

\begin{abstract}
We study the effect of the environment on the process of the measurement of a state of a microscopic spin half system. The measuring apparatus is a heavy particle, whose center of mass coordinates can be considered at the end of the measurement as approximately classical, and thus can be used as a pointer. The state of the pointer, which is the result of its interaction with the spin, is transformed into a mixed state by the coupling of the pointer to the environment. The environment is considered to be a gas reservoir, whose particles interact with the pointer. This results in a Fokker-Planck equation for the reduced density matrix of the pointer. The solution of the equation shows that the quantum coherences, which are characteristic to the entangled state between the probabilities to find the pointer in one of two positions, decays exponentially fast in time. We calculate the exponential decay function of this decoherence effect, and express it in terms of the parameters of the
\end{abstract}

\footnotetext{
*Corresponding author: kleckner@tx.technion.ac.il
} 
model.

PACS Numbers: 03.65.Bz

Typeset using REVTEX 


\section{INTRODUCTION}

In recent years there has been a considerable interest in the effect of the environment on

the process of quantum measurement. The measurement of physical variable of a microscopic system - a system which is described by quantum theory - is usually realized by coupling the system to a "classical meter". The meter itself does not have to be macroscopic, however some of its physical variables, say the position of its center of mass, can be considered as approximately classical. If these variables are chosen to be correlated with those of the system, their readings are related to the measurement of the microscopic properties of the system. When the measurement process is efficient there is one to one correspondence between the macroscopic readings of the meter and the microscopic quantities, which are set to be measured. A particle, whose center of mass position serves as a meter, will be identified as a pointer. A theory that starts from a model Hamiltonian, which includes only the system, the pointer, and the coupling between them, cannot account for the real measurement process. The reason is that the classical meter is significantly influenced, on the time scale of its motion, by the environment around it. Even though the dynamics of the combined entity - the system, the meter, and the environment - can be described by quantum mechanics, the environment (bath) plays a distinctive role in the process.

The role of the environment is formally expressed in the quantum description of the experiment in that the system ends up in a mixed state, rather then in a pure state, which is the outcome of a "pure" unitary transformation. While a unitary transformation leaves the entire entity, system, meter and bath, in an entangled state, so that the pointer's final positions are not definite, but rather display interference, the environment generates decoherence, and induces definite final readings of the meter. The fundamental nature of the quantum theory makes the predictions of the readings probabilistic, while the decoherence, which is caused by the nature of the environment, is responsible for the definite positions of the pointer.

That decoherence is induced by the environment was recognized long ago, as was pointed 
out explicitly almost twenty years ago by Zurek [1-3. This point was previously noticed by Zeh [4] and Kübler and Zeh [5] in the early seventies. Soon later Caldeira and Leggett [6,7], and Walls, Milburn and Collet [8.9] have shown, using a harmonic oscillator as their microscopic quantum system, to be measured, and an ensemble of many harmonic oscillators to model the environment, that indeed the environment introduces decoherence to the Density Matrix of the quantum system. In Ref. [6] an initial pure state of two spatially separated Gaussian wave-packets of the microscopic system - a harmonic oscillator - are turned by the bath, in an extremely short time, into a well defined mixed state of the two wave-packets. Ref. [8] starts from a pure state with a superposition of two separated pure coherent states of a harmonic oscillator, and ends up, again due to the interaction with the bath, in a mixed state of the two coherent states. The quantum interferences between the two wave-packets, or the two coherent states, fade away in an extremely short time. Kübler and Zeh [5] and later Zurek et al. [10 have shown that the coherent states of a harmonic oscillator in a bath are somewhat unique in their decoherence, being almost classical. Collett [11] has extended the investigation of decoherence using density-matrix calculations for some simple open systems. Following the ingenious experiments of the Ecole Normal Superieure Group [12] Paz and Zurek [13] have investigated the decoherence in the limit of weak interaction between the "meter" and the bath. Recently the decoherence of a harmonic oscillator, playing the role of a meter measuring a spin half system, has been studied by Venugopalan [14]. Their model for the bath was the standard one [6], namely an ensemble of harmonic oscillators.

In the present paper we revisit the decoherence problem, and study it more in the context the "measurement" theory in a somewhat different setup. We consider a simple model to describe a combined system, which is made of three coupled parts, a microscopic atomic system, a measuring apparatus, and a bath. While the standard model for the bath is an ensemble of independent harmonic oscillators, we consider here a gas reservoir, namely an ensemble of independent particles, which interact with the meter. The initial state of a spinhalf atom is set to be "measured" by the final position of a massive particle, which is, in turn, in contact with the reservoir. This combined system is supposed to simulate the entire 
process of a "real" measurement. An environment of a gas seems to be more appropriate for the description of the motion of a particle-pointer. Furthermore, by changing the density of the gas one can control the time of decoherence. The atom interacts for a very short time with the meter, and the state of the two systems is becoming entangled. The two macroscopically separated positions of the pointer, which are now quantum-mechanically correlated with the initial state of the spin, interfere with each other. The interaction of the meter with the particles of the bath introduces decoherence, namely is destroying the interference, and making the pointer's readings distinguishable. The development in time of the entire system is described by a unitary transformation, which originates probability into the dynamics by quantum mechanics. This evolution in time is conveniently described by the density-matrix of the entire system. In the treatment of the measurement process, a pure state is transformed after integrating out the environment into a mixed state, and the notion of the collapse of the wave function is never being introduced. The probabilistic nature of microscopic dynamics, which is built into the quantum description, is expressed, at the end of the process, in terms of the two potentially definite positions of the pointer.

The crucial role of the environment is clearly expressed in the stage where the densitymatrix of the combined system of the pointer and the bath is traced over the degrees of freedom of the gas particles of the reservoir. Since the exact initial conditions of the bath cannot be completely prescribed, the unitary transformation, which controls the motion in time, does not account for the complete description of the entire process. The point is that even if we could theoretically prescribe the state of the bath at a given time, it cannot be completely decisive for an actual experiment. The reason is that the rate of change of the environment states is order of magnitudes faster than the inverse time scales of the experiment. Since these time scales in reality are so different, even the notion of initial conditions is not well defined. Relevant initial conditions can be meaningfully prescribed, for two interacting systems, only when their intrinsic time scales are not orders of magnitudes apart. A theory, which is being introduced to study a real experiment, has to incorporate these facts into its structure. It is manifested here by assuming that the reservoir is in 
thermal equilibrium. This has nothing to do with the consistency of quantum theory, but rather with the role of the real initial conditions of the entity we set to investigate. It is our opinion that a theory, being a logical construction in terms of a mathematical scheme, is supposed to be correlated with the intrinsic approximate nature of the results of real experiments, and it has to reflect this facet of physical reality.

The outline of the paper is as follows: In Section II we introduce the model to be investigated in terms of the Hamiltonians for the spin-half atom, the massive pointer, the gas reservoir, and the interactions among them. Section III is concerned with the motion of the atom and the pointer without the reservoir. The quantum interference between the two final positions of the pointer is studied in detail. In Section IV we add the bath into the development in time, introduce the Fokker-Planck equation for the pointer in the gas environment, and show how the decoherence is set in. In Section V, we discuss the conclusions. The details of the solution of the Fokker-Planck equation for the Wigner distribution is outlined in Appendix A. In Appendix B we study an intuitive model for the bath, which is simulated by a random field perturbing the meter, and compare it with the results obtained for the gas reservoir.

\section{MODEL HAMILTONIAN}

We consider the measurement of the spin components of a spin half atom. We introduce, for our microscopic system $S$, i.e., the spin half atom, the Hamiltonian, $H_{S}$, which, in the absence of an external magnetic field, has degenerate energy eigenstates

$$
H_{S}| \pm 1\rangle=\epsilon_{0}| \pm 1\rangle \text {. }
$$

These are also eigenstates of $\sigma_{z}$, the component of the spin operator along the $z$-axis, i.e., $\sigma_{z}|\sigma\rangle=\sigma|\sigma\rangle$, where $\sigma= \pm 1$, for the up or down states. We shall take $\epsilon_{0}=0$.

Our measuring device, the pointer, is taken to be a massive particle of mass $M$ (possibly a heavy atom), whose center of mass position and momentum are $\mathbf{R}$ and $\mathbf{P}$ respectively. The Hamiltonian, $H_{P}$, of the pointer is then 


$$
H_{P}=\frac{P^{2}}{2 M} .
$$

The pointer is the meter which is assigned to perform a measurement of the $z$-components of the spin of the atom. A measurement is thought of as a process, which generates a correlation between the measured property of the microscopic system, i.e. the spin component, and say, the final macroscopic position of the pointer. To measure the spin variable $\sigma_{z}$, which has the observable outcomes \pm 1 , we adopt, after Peres [15], the following coupling Hamiltonian:

$$
H_{S P}=V(t) P_{x} \sigma_{z}
$$

where $V(t)$ is a c-number time dependent "impact" function, which couples the spin operator to the pointer. The function $V(t)$ has the dimension of velocity, and is active for a very short finite interaction time, $0 \leq t \leq T$. We wish to point out that one massive atom can play the role of both the pointer and the spin half system, when the internal microscopic variable - the spin - is being coupled for a short time with the center of mass of the atom, as in a Stern-Gerlach device for example.

The effect of the environment on the pointer is to be studied here assuming the following picture. The pointer is considered to be immersed in a bath, which is viewed as a set of independent identical "field" particles, whose $i$-th particle is described by the mass $m$, coordinate $\mathbf{r}_{i}$, and momentum $\mathbf{p}_{i}$, and the Hamiltonian

$$
H_{B}=\sum_{i} \frac{p_{i}^{2}}{2 m} .
$$

Each of these bath particles interacts with the pointer via a potential $\phi\left(\mathbf{R}-\mathbf{r}_{i}\right)$, and thus the pointer - bath interaction Hamiltonian is

$$
H_{P B}=\sum_{i} \phi\left(\mathbf{R}-\mathbf{r}_{i}\right)
$$

The total Hamiltonian for our system is thus

$$
H=H_{S}+H_{P}+H_{S P}+H_{B}+H_{P B} .
$$


The outline of the procedure employed for the measurement of the spin of the atom is as follows. Suppose that the atom is initially in the state

$$
\left|\psi_{S}(0)\right\rangle=\sum_{\sigma} a_{\sigma}|\sigma\rangle=a_{+1}|+1\rangle+a_{-1}|-1\rangle
$$

where $a_{\sigma}$ is the amplitude to find the spin in the $\sigma$ state, $a_{\sigma}^{*}$ is the complex conjugate of $a_{\sigma}$, and $\sum_{\sigma} a_{\sigma}^{*} a_{\sigma}=1$. This state is to be measured by the position of the pointer after it interacts with the atom. The initial state of the pointer, which is also described by quantum mechanics, is assumed to be a Gaussian wave packet:

$$
\psi(\mathbf{R}, 0)=\left(2 \pi \Delta^{2}\right)^{-3 / 4} e^{-R^{2} / 4 \Delta^{2}}
$$

where $\Delta$ is the spread of the wave packet in $R$, namely, the initial expectation value of $R$ is 0 , and that of $R^{2}$ is $\Delta^{2}$. We take $\Delta$ to be as small as possible, to have a better resolution of the position of the pointer. The change of the state of the pointer in time, due to its interaction with the atom and the environment, is now studied in details, by solving the

equation of motion of the pointer and eliminating the degrees of freedom of the "gas" of field particles surrounding the pointer. But before we will do that, let us first take a look at the case where there is no bath and the measurement of the spin is attempted with a "free" pointer.

\section{MEASURING THE SPIN BY A FREE POINTER}

First we consider our system without the environment, and find the time development of the pointer from its given initial conditions. Since the "free" spin term of the atom is irrelevant, the Hamiltonian is

$$
H=\frac{\mathbf{P}^{2}}{2 M}+V(t) P_{x} \sigma_{z}
$$

The state of the combined system, atom and pointer, can be given at the time $t$, by the wave function $\Psi(\mathbf{R}, \sigma, t)$, which is the solution of the Schrödinger's equation 


$$
i \hbar \dot{\Psi}(\mathbf{R}, \sigma, t)=\left(-\frac{\hbar^{2}}{2 M} \frac{\partial^{2}}{\partial \mathbf{R}^{2}}-i \hbar \sigma V(t) \frac{\partial}{\partial X}\right) \Psi(\mathbf{R}, \sigma, t)
$$

with the initial condition

$$
\Psi(\mathbf{R}, \sigma, 0)=a_{\sigma}\left(2 \pi \Delta^{2}\right)^{-3 / 4} e^{-R^{2} / 4 \Delta^{2}}
$$

It is convenient to introduce the momentum representation for the pointer, namely $\mathbf{P}|\mathbf{k}\rangle=$ $\hbar \mathbf{k}|\mathbf{k}\rangle$, and apply the Fourier transform to the wave function,

$$
\Psi(\mathbf{R}, \sigma, t)=\frac{1}{(2 \pi)^{3}} \int_{-\infty}^{+\infty} d^{3} k e^{+i \mathbf{k} \cdot \mathbf{R}} \Psi(\mathbf{k}, \sigma, t)
$$

The equation of motion takes the form

$$
i \hbar \frac{\partial}{\partial t} \Psi(\mathbf{k}, \sigma, t)=\left(\frac{\hbar^{2} k^{2}}{2 M}+V(t) \hbar k_{x} \sigma\right) \Psi(\mathbf{k}, \sigma, t)
$$

where $k=|\mathbf{k}|$, and the initial condition is given by

$$
\Psi(\mathbf{k}, \sigma, 0)=a_{\sigma}\left(8 \pi \Delta^{2}\right)^{3 / 4} e^{-\Delta^{2} k^{2}}
$$

The solution of Eq. (13) is then

$$
\Psi(\mathbf{k}, \sigma, t)=\Psi(\mathbf{k}, \sigma, 0) e^{-i \omega_{k} t-i \bar{X}(t) k_{x} \sigma}
$$

where $\omega_{k}=\frac{\hbar k^{2}}{2 M}$, and $\bar{X}(t)=\int_{0}^{t} d t^{\prime} V\left(t^{\prime}\right)$.

It is convenient to discuss the measurements in terms of the density matrix of the combined system. In the $(\mathbf{k}, \sigma)$ representation we write the density matrix as

$$
\rho\left(\mathbf{k}, \sigma ; \mathbf{k}^{\prime}, \sigma^{\prime} ; t\right)=\Psi^{*}\left(\mathbf{k}^{\prime}, \sigma^{\prime}, t\right) \Psi(\mathbf{k}, \sigma, t),
$$

where $\Psi^{*}$ is the complex conjugate of $\Psi$. From Eq. (15) we get

$$
\begin{aligned}
\rho\left(\mathbf{k}, \sigma ; \mathbf{k}^{\prime}, \sigma^{\prime} ; t\right)= & \rho\left(\mathbf{k}, \sigma ; \mathbf{k}^{\prime}, \sigma^{\prime} ; 0\right) \\
& \times e^{-i\left(\omega_{k}-\omega_{k^{\prime}}\right) t} e^{-i \bar{X}(t)\left(k_{x} \sigma-k_{x}^{\prime} \sigma^{\prime}\right)},
\end{aligned}
$$

where, using Eq. (14), the density matrix is initially 


$$
\rho\left(\mathbf{k}, \sigma ; \mathbf{k}^{\prime}, \sigma^{\prime} ; 0\right)=a_{\sigma^{\prime}}^{*} a_{\sigma}\left(8 \pi \Delta^{2}\right)^{3 / 2} e^{-\Delta^{2}\left(k^{2}+k^{\prime 2}\right)} .
$$

We now return to real space, and transform the density matrix to the $(\mathbf{R}, \sigma)$ representation to find:

$$
\begin{aligned}
\rho\left(\mathbf{R}, \sigma ; \mathbf{R}^{\prime}, \sigma^{\prime} ; t\right)= & a_{\sigma^{\prime}}^{*} a_{\sigma}\left(2 \pi \Delta^{2} \xi(t)\right)^{-3 / 2} \\
& \times \exp \left(-\frac{1}{4 \Delta^{2} \xi(t)}\left(\zeta^{*}(t)[\mathbf{R}-\hat{\mathbf{x}} \bar{X}(t) \sigma]^{2}+\zeta(t)\left[\mathbf{R}^{\prime}-\hat{\mathbf{x}} \bar{X}(t) \sigma^{\prime}\right]^{2}\right)\right),
\end{aligned}
$$

where $\zeta(t)=1+i t / \tau_{f}, \xi(t)=\zeta^{*}(t) \zeta(t)=1+\left(t / \tau_{f}\right)^{2}$. Here $\tau_{f}=2 M \Delta^{2} / \hbar$ is a characteristic time of free quantum diffusion of the pointer, and $\hat{\mathbf{x}}$ is a unit vector along the $x$ - axis. Eq. (19) is our result for the density matrix of the "free" pointer. In passing we notice that we can express formally the density matrix at time $t$ in terms of the density matrix at $t=0$, namely

$$
\rho\left(\mathbf{R}, \sigma ; \mathbf{R}^{\prime}, \sigma^{\prime} ; t\right)=\int_{-\infty}^{+\infty} d^{3} Y \int_{-\infty}^{+\infty} d^{3} Y^{\prime} J\left(\mathbf{R}, \mathbf{R}^{\prime}, t ; \mathbf{Y}, \mathbf{Y}^{\prime}, 0\right) \rho\left(\mathbf{Y}, \sigma ; \mathbf{Y}^{\prime}, \sigma^{\prime} ; 0\right)
$$

where the propagation kernel is

$$
\begin{aligned}
J\left(\mathbf{R}, \mathbf{R}^{\prime}, t ; \mathbf{Y}, \mathbf{Y}^{\prime}, 0\right) & =\frac{1}{(2 \pi)^{6}} \int_{-\infty}^{+\infty} d^{3} k^{\prime} e^{-i \mathbf{k}^{\prime} \cdot\left(\mathbf{R}^{\prime}-\mathbf{Y}^{\prime}\right)} \int_{-\infty}^{+\infty} d^{3} k e^{i \mathbf{k} \cdot(\mathbf{R}-\mathbf{Y})} e^{-i\left(\omega_{k}-\omega_{k^{\prime}}\right) t-i \bar{X}(t)\left(k_{x} \sigma-k_{x}^{\prime} \sigma^{\prime}\right)} \\
& =\left(\frac{M}{2 \pi \hbar t}\right)^{3}\left[e^{i M[\mathbf{R}-\mathbf{Y}-\hat{\mathbf{x}} \bar{X}(t) \sigma]^{2} / 2 \hbar t} e^{-i M\left[\mathbf{R}^{\prime}-\mathbf{Y}^{\prime}-\hat{\mathbf{x}} \bar{X}(t) \sigma^{\prime}\right]^{2} / 2 \hbar t}\right]
\end{aligned}
$$

We also wish to point out that we could have obtained Eq. (19) directly from Eq. (15) using

$$
\begin{aligned}
\Psi(\mathbf{R}, \sigma, t)= & \frac{1}{(2 \pi)^{3}} \int_{-\infty}^{+\infty} d^{3} k e^{-i \mathbf{k}^{\prime} \mathbf{R}^{\prime}} \Psi(\mathbf{k}, \sigma, t) \\
= & a_{\sigma}\left(2 \pi \Delta^{2} \zeta^{2}(t)\right)^{-3 / 4} \\
& \times \exp \left(-\frac{1}{4 \Delta^{2} \zeta(t)}[\mathbf{R}-\hat{\mathbf{x}} \bar{X}(t) \sigma]^{2}\right),
\end{aligned}
$$

and $\rho\left(\mathbf{R}, \sigma ; \mathbf{R}^{\prime}, \sigma^{\prime} ; t\right)=\Psi^{*}\left(\mathbf{R}^{\prime}, \sigma^{\prime}, t\right) \Psi(\mathbf{R}, \sigma, t)$.

We now turn to study Eq. (19). First we observe that our density matrix represents a pure state. It is just the evolution in time, by a unitary transformation of the initial product 
state, $\Psi(0)=\left(a_{+1}+a_{-1}\right) \psi(\mathbf{R}, 0)$, into an entangled state of the particle and the pointer, namely

$$
\Psi(t)=a_{+1} \psi_{+}(\mathbf{R}, t)+a_{-1} \psi_{-}(\mathbf{R}, t)
$$

where

$$
\begin{aligned}
\psi_{ \pm}(\mathbf{R})= & \left(2 \pi \Delta^{2} \zeta^{2}(t)\right)^{-3 / 4} \\
& \times \exp \left(-\frac{1}{4 \Delta^{2} \zeta(t)}[\mathbf{R} \mp \hat{\mathbf{x}} \bar{X}(t)]^{2}\right)
\end{aligned}
$$

are two displaced wave packets of the pointer. In this way the amplitude of the position of the pointer is correlated to the spin of the particle. This is not yet a measurement, but rather an establishment of quantum mechanical correlations, due to the interaction between the "free" pointer and the particle.

The outcome of a measurement of the position of the pointer is then predicted, using the combined system's density matrix, by calculating the probability density to detect the center of mass of the pointer around $\mathbf{R}$, i.e., $P(\mathbf{R}, t)=\sum_{\sigma, \sigma^{\prime}} \rho\left(\mathbf{R}, \sigma ; \mathbf{R}, \sigma^{\prime} ; t\right)$. Since the measurement is done after the interaction was completed, namely at a time $t>T$, we set $\bar{X}(t)=\bar{X}=\int_{0}^{T} d t^{\prime} V\left(t^{\prime}\right)$, and write $P(\mathbf{R}, t)$ as a sum of three terms:

$$
P(\mathbf{R}, t)=P_{+1}(\mathbf{R}, t)+P_{-1}(\mathbf{R}, t)+P_{+1,-1}(\mathbf{R}, t)
$$

Using $\Delta_{f}^{2}(t)=\Delta^{2} \xi(t)$, the first term, with $\sigma=\sigma^{\prime}=+1$, is

$$
\begin{aligned}
P_{+1}(\mathbf{R}, t)= & \left|a_{+1}\right|^{2}\left(2 \pi \Delta_{f}^{2}(t)\right)^{-3 / 2} \\
& \times \exp \left(-\frac{1}{2 \Delta_{f}^{2}(t)}[\mathbf{R}-\hat{\mathbf{x}} \bar{X}]^{2}\right),
\end{aligned}
$$

and second term, with $\sigma=\sigma^{\prime}=-1$, is

$$
\begin{aligned}
P_{-1}(\mathbf{R}, t)= & \left|a_{-1}\right|^{2}\left(2 \pi \Delta_{f}^{2}(t)\right)^{-3 / 2} \\
& \times \exp \left(-\frac{1}{2 \Delta_{f}^{2}(t)}[\mathbf{R}+\hat{\mathbf{x}} \bar{X}]^{2}\right),
\end{aligned}
$$


corresponding to the probabilities of up and down spins. While the third term, with $a_{ \pm 1}=$ $\left|a_{ \pm 1}\right| e^{i \varphi_{ \pm}}$, is

$$
\begin{aligned}
P_{+1,-1}(\mathbf{R}, t)= & 2 \sqrt{P_{+}(\mathbf{R}, t) P_{-}(\mathbf{R}, t)} \\
& \times \cos \left(\frac{\bar{X} X}{\Delta_{f}^{2}(t)} \frac{t}{\tau_{f}}+\varphi_{-}-\varphi_{+}\right),
\end{aligned}
$$

is due to quantum interference between the two spin states. In Eqs. (26), (27) and (28)

$$
\Delta_{f}^{2}(t)=\Delta^{2}\left[1+\left(t / \tau_{f}\right)^{2}\right]
$$

reflects the spatial broadening of the pointer's position as a result of the free quantum diffusion. At this point we note that if the "pointer" is a Silver atom, with a mass of $M=1.8 \times 10^{-22} \mathrm{~g}$, and initial spread of position $\Delta=1 \mu \mathrm{m}$,

$$
\tau_{f}=\frac{2 M \Delta^{2}}{\hbar} \simeq 3 \mathrm{~ms}
$$

which is a long time on the scale of a ballistic experiment of an atom.

The probability distribution for the position of the pointer, long after it departed from the "measured" particle, has three components: two of them are "normal", and the third one is "strange". As it is clear from Eqs. (26) and (27), one component represents a positive deflection of the pointer by $+\bar{X}$, and displays a Gaussian centered around $(+\bar{X}, 0,0)$, which is correlated with the spin up state. The other one indicates a negative deflection by $-\bar{X}$, and displays a Gaussian centered around $(-\bar{X}, 0,0)$, which is correlated with the spin down state of the measured system. The width of each term is of the order of the spread of the initial distribution. However the weight of each peek, i.e., the probability of positive or negative deflection, is decided by the initial probability of the two spin states, namely by $\left|a_{\sigma}\right|^{2}$. The probability distribution of Eq. (28) exhibits an interference between the two displaced Gaussians. Notice that this interference term oscillates in time with a frequency given by

$$
\Omega_{i n t}=\frac{\bar{X} X}{\Delta^{2}} \frac{1}{\tau_{f}} .
$$


This interference is an unambiguous outcome of the quantum mechanical development in time of the pure entangled state of the pointer and the system to be measured. This result is completely reversible in time, and in principle can be reversed to the initial setup.

\section{THE POINTER IN A BATH}

We turn now to consider the case in which, in addition to the "external" interaction with the spin, the pointer is coupled to a reservoir. Our task is to describe the evolution in time of the pointer's state under the influence of this reservoir. To this end, we will drive a master equation for the reduced density matrix $\rho(t)$ of the pointer, following the method outlined in Ref. [16]. The main ingredients of this method are as follows: The effect of the pointer on the bath is assumed to be very small, and the interaction $H_{P B}$ is considered as a weak perturbation. The equation of motion for the density matrix of the combined system, pointer and bath, is written up to second order in $H_{P B}$. Finally, by tracing over the degrees of freedom of the bath, one obtains an equation of motion for the pointer only, which is valid on time scales much larger compared with the typical correlation times of the bath. We choose here a particular model for the bath, which is constituted of a set of independent

field particles, and trace over the dynamical variables of these particles. This results in a Fokker - Planck equation for the reduced density matrix, which can then be solved for the time dependent behavior of the pointer.

\section{A. The Reduced Density Matrix}

In order to study the effect of the interaction, $H_{P B}$, on the pointer, it will be convenient to introduce the Fourier transform of the interaction potential, $\phi(\mathbf{r})$, in space, in a volume $V:$

$$
\phi(\mathbf{r})=\frac{1}{V} \sum_{\mathbf{q}} e^{i \mathbf{q} \cdot \mathbf{r}} \phi(\mathbf{q}),
$$

Then the interaction Hamiltonian can be written as 


$$
H_{P B}=\frac{1}{V} \sum_{\mathbf{q}} e^{i \mathbf{q} \cdot \mathbf{R}} \phi(\mathbf{q}) n(\mathbf{q})
$$

where the Fourier transform of the density (operator) of the field particles is

$$
n(\mathbf{q})=\sum_{i} e^{-i \mathbf{q} \cdot \mathbf{r}_{i}}
$$

To get the equation of motion for the reduced density matrix for the pointer, we transform to the Interaction Representation (IR), and write the Hamiltonian as

$$
H_{P B}(t)=\frac{1}{V} \sum_{\mathbf{q}} \phi(\mathbf{q}) n(\mathbf{q}, t) A_{\mathbf{q}}(t)
$$

where, for the pointer

$$
A_{\mathbf{q}}(t)=e^{i H_{p} t / \hbar} e^{i \mathbf{q} \cdot \mathbf{R}} e^{-i H_{p} t / \hbar}
$$

and for the bath operator

$$
n(\mathbf{q}, t)=e^{i H_{B} t / \hbar} n(\mathbf{q}) e^{-i H_{B} t / \hbar} .
$$

The reduced density matrix of the pointer, in the IR,

$$
\bar{\rho}(t)=\operatorname{Tr}_{B}\left\{e^{i\left(H_{P}+H_{B}\right) t / \hbar} \rho(t) e^{-i\left(H_{P}+H_{B}\right) t / \hbar}\right\}
$$

obeys the following equation, [16] Eq. (B.30):

$$
\begin{aligned}
\frac{\Delta \bar{\rho}(t)}{\Delta t}=- & \frac{1}{\hbar^{2}} \frac{1}{\Delta t} \int_{t}^{t+\Delta t} d t_{1} \int_{t}^{t_{1}} d t_{2} \frac{1}{V} \sum_{\mathbf{q}} \phi(\mathbf{q}) \frac{1}{V} \sum_{\mathbf{q}^{\prime}} \phi\left(\mathbf{q}^{\prime}\right) \\
& \operatorname{Tr}_{b}\left\{\left[n\left(\mathbf{q}, t_{1}\right) A_{\mathbf{q}}\left(t_{1}\right),\left[n\left(\mathbf{q}^{\prime}, t_{2}\right) A_{\mathbf{q}^{\prime}}\left(t_{2}\right), \bar{\rho}(t) \otimes \rho_{B}\right]\right\}\right.
\end{aligned}
$$

where the trace is taken over the field particles' degrees of freedom, and $\rho_{B}$ is the reduced density matrix of the bath. This master equation, Eq. (39) is similar to Eq.(1) of Paz and Zurek [13], and to that of Unruh and Zurek [17]; however unlike the scalar potential that simulates their bath, here the environment is a gas. Another general master equation was derived by Joos 18 following Pauli, without specifying the bath. 
Assuming that the distribution of the field particles is stationary in time, and homogeneous in space, we can express the trace of the RHS of Eq.(39) in terms of the density density correlation function (CF) of the bath,

$$
\begin{aligned}
\operatorname{Tr}_{B}\left\{\rho_{B} n(\mathbf{q}, t+\tau) n\left(\mathbf{q}^{\prime}, t\right)\right\} & =\left\langle n(\mathbf{q}, t+\tau) n\left(\mathbf{q}^{\prime}, t\right)\right\rangle \\
& =\delta_{\mathbf{q}^{\prime},-\mathbf{q}} g_{\mathbf{q}}(\tau), \\
g_{\mathbf{q}}(\tau) & =\langle n(\mathbf{q}, t+\tau) n(-\mathbf{q}, t)\rangle .
\end{aligned}
$$

Eq. (39) using (40) is written as [16] Eq. (B.33).

$$
\begin{aligned}
\frac{\Delta \bar{\rho}(t)}{\Delta t}= & -\frac{1}{\hbar^{2}} \frac{1}{V^{2}} \sum_{\mathbf{q}}|\phi(\mathbf{q})|^{2} \int_{0}^{\infty} d \tau \frac{1}{\Delta t} \int_{t}^{t+\Delta t} d t^{\prime} \\
& \times\left\{g_{\mathbf{q}}(\tau)\left(A_{\mathbf{q}}\left(t^{\prime}\right) A_{-\mathbf{q}}\left(t^{\prime}-\tau\right) \bar{\rho}(t)-A_{-\mathbf{q}}\left(t^{\prime}-\tau\right) \bar{\rho}(t) A_{\mathbf{q}}\left(t^{\prime}\right)\right)\right. \\
& \left.+g_{-\mathbf{q}}(-\tau)\left(\bar{\rho}(t) A_{-\mathbf{q}}\left(t^{\prime}-\tau\right) A_{\mathbf{q}}\left(t^{\prime}\right)-A_{\mathbf{q}}\left(t^{\prime}\right) \bar{\rho}(t) A_{-\mathbf{q}}\left(t^{\prime}-\tau\right)\right)\right\} .
\end{aligned}
$$

We further assume that the reservoir is in thermal equilibrium in temperature $T$, and thus $\rho_{B}=e^{-\beta H_{B}} / Z$, where $\beta=1 / k_{B} T$ is the inverse temperature, $k_{B}$ is the Boltzmann factor, and $Z$ is the canonical partition function. Introducing the Fourier transform in time of the $\mathrm{CF}$,

$$
g_{\mathbf{q}}(t)=\frac{1}{2 \pi} \int_{-\infty}^{\infty} d \omega e^{-i \omega t} g_{\mathbf{q}}(\omega),
$$

we can write the spectral density of the CF as

$$
\begin{aligned}
g_{\mathbf{q}}(\omega)= & \frac{2 \pi \hbar}{Z} \sum_{\mu} e^{-\beta E_{\mu}} \sum_{\nu}\langle\mu|n(\mathbf{q})| \nu\rangle\langle\nu|n(-\mathbf{q})| \mu\rangle \\
& \times \delta\left(E_{\mu}-E_{\nu}+\hbar \omega\right),
\end{aligned}
$$

in terms of the eigenstates of $H_{B}$, i.e., $H_{B}|\mu\rangle=E_{\mu}|\mu\rangle$. First we Notice that since $n(-\mathbf{q})$ is the Hermitian conjugate of $n(\mathbf{q}), g_{\mathbf{q}}(\omega)$ is a real function of $\mathbf{q}, \omega$. Then we observe that $g_{-\mathbf{q}}(-\omega)=e^{-\beta \hbar \omega} g_{\mathbf{q}}(\omega)$, which is a statement of the detail balance induced by the thermal reservoir on the states of the pointer.

We consider a simple model for the bath, namely a gas of non-interacting particles in temperature $T$, and average density $n_{0}$. The mass $m$ of the bath particles is taken to 
be much smaller than that of the pointer, i.e., $m / M \ll 1$. To calculate the correlation function we first express the density operator, $n(\mathbf{q})=\sum_{\mathbf{p}} a_{\mathbf{p}-\mathbf{q}}^{\dagger} a_{\mathbf{p}}$, in terms $a_{\mathbf{p}}^{\dagger}$ and $a_{\mathbf{p}}$, the creation and annihilation operators of a "field" particle in the momentum eigenstate $|\mathbf{p}\rangle$, of energy $\epsilon_{\mathbf{p}}=\hbar^{2} p^{2} / 2 m$, and momentum $\hbar \mathbf{p}$. Since in the interaction representation $a_{\mathbf{p}}(t)=a_{\mathbf{p}} \exp \left(-i \omega_{\mathbf{p}} t\right)$, where $\epsilon_{\mathbf{p}}=\hbar \omega_{\mathbf{p}}$, and in thermal equilibrium $\left\langle a_{\mathbf{p}-\mathbf{q}}^{\dagger} a_{\mathbf{p}} a_{\mathbf{p}^{\prime}+\mathbf{q}}^{\dagger} a_{\mathbf{p}^{\prime}}\right\rangle=$ $\delta_{\mathbf{p}^{\prime}, \mathbf{p}-\mathbf{q}} f_{\mathbf{p}-\mathbf{q}}\left(1 \pm f_{\mathbf{p}}\right)$, where \pm is for either Bose or Fermi particles, and $f_{\mathbf{p}}$ stands for BoseEinstein of Fermi-Dirac distributions, the correlation function is then

$$
g_{\mathbf{q}}(\tau)=\sum_{\mathbf{p}} f_{\mathbf{p}-\mathbf{q}}\left(1 \pm f_{\mathbf{p}}\right) e^{-i\left(\omega_{\mathbf{p}}-\omega_{\mathbf{p}-\mathbf{q}}\right) \tau}
$$

and its spectral density is

$$
g_{\mathbf{q}}(\omega)=2 \pi \sum_{\mathbf{p}} f_{\mathbf{p}}\left(1 \pm f_{\mathbf{p}+\mathbf{q}}\right) \delta\left(\left(\omega_{\mathbf{p}+\mathbf{q}}-\omega_{\mathbf{p}}\right)-\omega\right),
$$

We now return to Eq.(41), and express the reduced density matrix for the pointer in its momentum representation. We denote by $|\mathbf{k}\rangle$, the eigenstate of momentum $\hbar \mathbf{k}$, and energy $E_{\mathbf{k}}=\hbar^{2} k^{2} / 2 M$, and find for the matrix elements of the pointer's operator $A_{\mathbf{q}}(t)$ in the IR

$$
\begin{aligned}
& \left\langle\mathbf{k}\left|A_{\mathbf{q}}(t)\right| \mathbf{k}^{\prime}\right\rangle=\left\langle\mathbf{k}\left|e^{i H_{B} t / \hbar} e^{i \mathbf{q} \cdot \mathbf{R}} e^{-i H_{B} t / \hbar}\right| \mathbf{k}^{\prime}\right\rangle \\
& =e^{i \Omega_{\mathbf{k}, \mathbf{k}^{\prime}} t} \delta_{\mathbf{k}^{\prime}, \mathbf{k}-\mathbf{q}}
\end{aligned}
$$

where $\Omega_{\mathbf{k}, \mathbf{k}^{\prime}}=\left(E_{\mathbf{k}}-E_{\mathbf{k}^{\prime}}\right) / \hbar$. In the Secular Approximation the equation of motion for the reduced density matrix, in the Schrödinger Picture can be written as

$$
\frac{d}{d t}\left\langle\mathbf{k}|\rho(t)| \mathbf{k}^{\prime}\right\rangle-\frac{1}{i \hbar}\left\langle\mathbf{k}\left|\left[H_{p}, \rho(t)\right]\right| \mathbf{k}^{\prime}\right\rangle=I_{\mathbf{k}, \mathbf{k}^{\prime}}(t) .
$$

Here the effect of the environment is given by the collision term

$$
\begin{aligned}
I_{\mathbf{k}, \mathbf{k}^{\prime}}(t)= & -\frac{1}{\hbar^{2}} \frac{1}{V^{2}} \sum_{\mathbf{q}}|\phi(\mathbf{q})|^{2} \\
& \times\left\{\left(G_{\mathbf{q}}\left(\Omega_{\mathbf{k}, \mathbf{k}-\mathbf{q}}\right)+G_{\mathbf{q}}^{*}\left(\Omega_{\mathbf{k}^{\prime}, \mathbf{k}^{\prime}-\mathbf{q}}\right)\right)\left\langle\mathbf{k}|\rho(t)| \mathbf{k}^{\prime}\right\rangle\right. \\
& \left.-\left(G_{\mathbf{q}}\left(\Omega_{\mathbf{k}+\mathbf{q}, \mathbf{k}}\right)+G_{\mathbf{q}}^{*}\left(\Omega_{\mathbf{k}^{\prime}+\mathbf{q}, \mathbf{k}^{\prime}}\right)\right)\left\langle\mathbf{k}+\mathbf{q}|\rho(t)| \mathbf{k}^{\prime}+\mathbf{q}\right\rangle\right\},
\end{aligned}
$$

where 


$$
G_{\mathbf{q}}(\omega)=\int_{0}^{\infty} d \tau e^{i \omega \tau} g_{\mathbf{q}}(\tau)
$$

is the one-sided Fourier transform of the correlation function. If we discard the frequency shifts due to the bath, we can replace in the above equation $G_{\mathbf{q}}(\omega)$ by the real function $g_{\mathbf{q}}(\omega) / 2$. To further facilitate the $\mathbf{p}$-summation in the $g_{\mathbf{q}}(\omega)^{\prime} s$, we make use of the $\delta-$ functions, and write, for example

$$
\begin{aligned}
G_{\mathbf{q}}\left(\Omega_{\mathbf{k}, \mathbf{k}-\mathbf{q}}\right)= & \frac{\pi m}{h^{2} q} \frac{1}{V} \sum_{\mathbf{p}} f_{\mathbf{p}}\left(1 \pm f_{\mathbf{p}+\mathbf{q}}\right) \\
& \times \delta\left\{\mathbf{p} \cdot \widehat{\mathbf{q}}-Q_{+}(\mathbf{k}, \mathbf{q})\right\}
\end{aligned}
$$

where $\hat{\mathbf{q}}=\mathbf{q} / q$, and

$$
Q_{ \pm}(\mathbf{k}, \mathbf{q})=\frac{m}{M} \widehat{\mathbf{q}} \cdot \mathbf{k}-\frac{q}{2}\left(1 \pm \frac{m}{M}\right)
$$

\section{B. Classical Gas Bath}

We now consider the bath as a classical gas, where the thermal distribution of the particles is Maxwellian, namely

$$
f_{\mathbf{p}}=n_{0}\left(\frac{\alpha}{\pi}\right)^{3 / 2} e^{-\alpha p^{2}}
$$

with $\alpha=\hbar^{2} / 2 m k_{B} T$. In this case, we have $f_{\mathbf{p}} \ll 1$, and if we replace the summation in Eq.(50) by integration over $\mathbf{p}$, i.e. write $\frac{1}{V} \sum_{\mathbf{p}} \Rightarrow \int d^{3} p /(2 \pi)^{3}$, we find

$$
G_{\mathbf{q}}\left(\Omega_{\mathbf{k}, \mathbf{k}-\mathbf{q}}\right)=\frac{1}{(2 \pi)^{3}} n_{0} \sqrt{\frac{\alpha}{\pi}} \frac{\pi m}{q \hbar^{2}} e^{-\alpha Q_{+}^{2}(\mathbf{k}, \mathbf{q})}
$$

and similarly for the other $G_{\mathbf{q}}$ 's of Eq.(48). The collision term can be then cast into

$$
\begin{aligned}
I_{\mathbf{k}, \mathbf{k}^{\prime}}(t)= & -\frac{1}{V} \sum_{\mathbf{q}}|\phi(\mathbf{q})|^{2} \frac{2 \pi}{\hbar} \frac{1}{(2 \pi)^{3}} n_{0} \sqrt{\frac{\alpha}{\pi}} \frac{m}{q \hbar^{2}} \\
& \times\left\{\left(e^{-\alpha Q_{+}^{2}(\mathbf{k}, \mathbf{q})}+e^{-\alpha Q_{+}^{2}\left(\mathbf{k}^{\prime}, \mathbf{q}\right)}\right) \times\left\langle\mathbf{k}|\rho(t)| \mathbf{k}^{\prime}\right\rangle\right. \\
& \left.-\left(e^{-\alpha Q_{-}^{2}(\mathbf{k}, \mathbf{q})}+e^{-\alpha Q_{-}^{2}\left(\mathbf{k}^{\prime}, \mathbf{q}\right)}\right) \times\left\langle\mathbf{k}+\mathbf{q}|\rho(t)| \mathbf{k}^{\prime}+\mathbf{q}\right\rangle\right\}
\end{aligned}
$$


This is still an exact result for a classical gas interacting with the pointer.

We now make use of the two small parameters in the problem, namely, that the mass ratio is small, and that the momentum transfer is small,

$$
\eta=m / M \ll 1 \text { and } q \ll k \text {. }
$$

First we expand $Q^{2}$ in small $\eta$. From Eq. (51) we have $Q_{ \pm}^{2}(\mathbf{k}, \mathbf{q}) \Rightarrow \frac{q^{2}}{4}-\eta\left(\mathbf{k} \cdot \mathbf{q} \mp \frac{q^{2}}{2}\right)+O\left(\eta^{2}\right)$, and thus, to first order in $\eta$ we can write

$$
e^{-\alpha Q_{ \pm}^{2}(\mathbf{k}, \mathbf{q})} \rightarrow e^{-\alpha \frac{q^{2}}{4}}\left(1+\alpha \eta\left(\mathbf{k} \cdot \mathbf{q} \mp \frac{q^{2}}{2}\right)\right)
$$

Then we expand $\left\langle\mathbf{k}+\mathbf{q}|\rho(t)| \mathbf{k}^{\prime}+\mathbf{q}\right\rangle$ in power series of $\mathbf{q}$, to obtain, up to second order in $q / k$

$$
\begin{aligned}
\left\langle\mathbf{k}+\mathbf{q}|\rho(t)| \mathbf{k}^{\prime}+\mathbf{q}\right\rangle= & \left\langle\mathbf{k}|\rho(t)| \mathbf{k}^{\prime}\right\rangle+\mathbf{q} \cdot\left(\frac{\partial}{\partial \mathbf{k}}+\frac{\partial}{\partial \mathbf{k}^{\prime}}\right)\left\langle\mathbf{k}|\rho(t)| \mathbf{k}^{\prime}\right\rangle \\
& +\frac{1}{2} \mathbf{q} \cdot\left(\frac{\partial}{\partial \mathbf{k}}+\frac{\partial}{\partial \mathbf{k}^{\prime}}\right) \mathbf{q} \cdot\left(\frac{\partial}{\partial \mathbf{k}}+\frac{\partial}{\partial \mathbf{k}^{\prime}}\right)\left\langle\mathbf{k}|\rho(t)| \mathbf{k}^{\prime}\right\rangle
\end{aligned}
$$

$>$ From the structure of this expansion we realize that it is convenient to express the density matrix in terms of the vectors $\mathbf{K}=\left(\mathbf{k}+\mathbf{k}^{\prime}\right) / 2$, and $\mathbf{p}=\left(\mathbf{k}-\mathbf{k}^{\prime}\right)$, namely write $\left\langle\mathbf{k}|\rho(t)| \mathbf{k}^{\prime}\right\rangle=\rho\left(\mathbf{k}, \mathbf{k}^{\prime}, t\right)=\rho(\mathbf{K}, \mathbf{p}, t)$. Substituting these expressions in Eq.(54), assuming that $\phi(\mathbf{q})$ is isotropic in $\mathbf{q}$, and integrating over $\mathbf{q}$, the collision term is simply

$$
\begin{aligned}
I_{\mathbf{k}, \mathbf{k}^{\prime}}(t)= & \gamma \frac{\partial}{\partial \mathbf{K}} \cdot(\mathbf{K} \rho(\mathbf{K}, \mathbf{p}, t)) \\
& +D \frac{\partial}{\partial \mathbf{K}} \cdot \frac{\partial}{\partial \mathbf{K}} \rho(\mathbf{K}, \mathbf{p}, t)
\end{aligned}
$$

Here

$$
\gamma=n_{0} \eta \frac{1}{(2 \pi)^{4}} \sqrt{\frac{\alpha}{\pi}} \frac{4 m \alpha}{3 \hbar^{3}} \int_{0}^{\infty} d q q^{3}|\phi(q)|^{2} e^{-\alpha \frac{q^{2}}{4}}
$$

plays the role of inverse relaxation time, and $D=\gamma / 2 \alpha \eta$ plays the role of a diffusion coefficient in $k$ space. Using [19] we can relate the $k$-diffusion coefficient, $D$, in Eq. (58) to the standard coefficient of spatial diffusion, $D_{c}=k_{B} T / M \gamma$, i.e., we have $D=(M \gamma / \hbar)^{2} D_{c}$. 
Notice that it is spatial diffusion coefficient, $D_{c}$, which is connected by the Einstein relation to $\gamma$.

The equation of motion of density matrix of the pointer, Eq. (47), becomes, with Eq. (58), a Fokker - Planck (FP) equation for $\left\langle\mathbf{k}|\rho(t)| \mathbf{k}^{\prime}\right\rangle$, namely

$$
\begin{aligned}
\frac{\partial}{\partial t}\left\langle\mathbf{k}|\rho(t)| \mathbf{k}^{\prime}\right\rangle-\frac{1}{i \hbar}\left\langle\mathbf{k}\left|\left[H_{P}, \rho(t)\right]\right| \mathbf{k}^{\prime}\right\rangle= & \gamma \frac{\partial}{\partial \mathbf{K}} \cdot\left(\mathbf{K}\left\langle\mathbf{k}|\rho(t)| \mathbf{k}^{\prime}\right\rangle\right) \\
& +D \frac{\partial}{\partial \mathbf{K}} \cdot \frac{\partial}{\partial \mathbf{K}}\left\langle\mathbf{k}|\rho(t)| \mathbf{k}^{\prime}\right\rangle .
\end{aligned}
$$

This equation is much like the FP equation derived by Caldeira and Lggett [7] for a harmonic oscillator interacting with a bath of harmonic oscillators in equilibrium. The main difference is that the friction parameter $\gamma$, here is given in terms of the properties of the gas surrounding the pointer.

Before we turn to the solution of this equation in time, we make some estimates of the Fokker-Planck coefficient, $\gamma$. If the interaction potential $\phi(\mathbf{r})$ is specified, we can calculate explicitly $\gamma$ of Eq.(59). Let us propose a particular interaction potential that will simulate the collisions between the pointer and the gas particles. We take

$$
\phi(\mathbf{r})=\phi_{0} e^{-r^{2} / a^{2}}
$$

where $a$ is the length scale of the pointer, and $\phi_{0}$ is the potential strength. Using this potential, the term $|\phi(q)|^{2}$ in Eq. (59) can be specified as

$$
|\phi(q)|^{2}=\left|\int \phi(\mathbf{r}) e^{-i \mathbf{q} \cdot \mathbf{r}} d \mathbf{r}\right|^{2}=\pi^{3} a^{6} \phi_{0}^{2} e^{-a^{2} q^{2} / 2} .
$$

For the inverse relaxation time we get

$$
\gamma=n_{0} \eta \sqrt{\frac{\alpha}{\pi^{3}}} \frac{m}{3 \hbar^{3}}\left(\phi_{0} a^{2}\right)^{2} \frac{\varrho}{(1+\varrho)^{2}},
$$

where $\varrho=2 a^{2} / \alpha$. Recalling that $\alpha=\hbar^{2} / 2 m k_{B} T$, and noting that even for objects with atomic dimensions $\varrho \gg 1$, we can write $\gamma$ as

$$
\gamma=\frac{1}{16} \sqrt{\frac{3}{2 \pi^{3}}} n_{0} \eta a^{2} \bar{v}\left(\frac{\phi_{0}}{\epsilon_{T}}\right)^{2}
$$


where $\epsilon_{T}=3 k_{B} T / 2$ is the average thermal energy and $\bar{v}=\sqrt{3 k_{B} T / m}$ is the average velocity of the gas particles. Although we have an explicit expression for $\gamma$, it is still necessary to specify $\phi_{0}$. For air in room temperature, a pointer which is a silver atom, we take $a=$ $1.75 \times 10^{-8} \mathrm{~cm}$ to be the radius of the atom, and $M=1.8 \times 10^{-22} \mathrm{~g}$. Assuming that the barrier $\phi_{0}$ is, say 50 times the thermal energy $\epsilon_{T}$, we get a typical $\gamma \simeq 2.5 \times 10^{9} \mathrm{~s}^{-1}$.

\section{Solution of the Fokker-Planck Equation for the Pointer in a Bath.}

In order to solve Eq. (60) for the pointer model outlined in section II, we further make two simplifying assumptions. First, we observe that since the deflection of the pointer as a result of the interaction with the spin is only along the $x$-axis, the coordinates $y$ and $z$ are of little consequence for our purpose. Hence we will consider only the one dimensional FP equation along the $x$-coordinate, and use, for convenience, $x$ instead of the $X$-coordinate of the pointer. If we set $\mathbf{k} \rightarrow k, \mathbf{k}^{\prime} \rightarrow k^{\prime}, K=\left(k+k^{\prime}\right) / 2$, and $p=\left(k-k^{\prime}\right)$ we can restrict ourselves only to

$$
\left\langle k|\rho(t)| k^{\prime}\right\rangle=\rho\left(k, k^{\prime}, t\right)=\rho(K, p, t) .
$$

Our second assumption is that the interaction of the pointer with the atom occurs in a very short time, or takes place before the interaction with the bath is switched on. In other words, we start from a density matrix which describes a free pointer after its interaction with the spin has already been completed, that is we use Eq. (17) as the initial condition for the FP equation for $\rho(K, p, t)$. It is clear that both assumptions are not necessary in order to carry out the calculation. The first one comes to simplify the mathematics by removing the irrelevant degrees of freedom, while the second one saves us the need to define $V(t)$ explicitly in Eq. (3). We bare in mind of course, that what we are really interested in here, is the decoherence process itself, and not so much the creation of the initial coherent state of the pointer.

Suppose therefore that we know the initial density matrix, $\rho(p, K, 0)$, and we seek the 
solution of $\rho(p, K, t)$ at the time $t$. The free streaming term of Eq. (60) for the pointer in its momentum representation is then

$$
\begin{aligned}
-\frac{1}{i \hbar}\left\langle k\left|\left[H_{P}, \rho(t)\right]\right| k^{\prime}\right\rangle & =-\frac{1}{i \hbar}\left(E_{k}-E_{k^{\prime}}\right) \rho\left(k, k^{\prime}, t\right) \\
& =i \hbar \frac{K p}{M} \rho(p, K, t),
\end{aligned}
$$

Inserting this back into Eq. (60) gives us

$$
\begin{aligned}
\frac{\partial}{\partial t} \rho(p, K, t)+i \hbar \frac{K p}{M} \rho(p, K, t)= & \gamma \frac{\partial}{\partial K}(K \rho(p, K, t)) \\
& +D \frac{\partial^{2}}{\partial K^{2}} \rho(p, K, t) .
\end{aligned}
$$

where the initial condition can be written with the aid of (17) and (18) as

$$
\begin{aligned}
\rho\left(p, K ; \sigma, \sigma^{\prime}, 0\right)= & a_{\sigma^{\prime}}^{*} a_{\sigma} 2\left(2 \pi \Delta^{2}\right)^{1 / 2} e^{-2 \Delta^{2} K^{2}-i K \bar{X}\left(\sigma-\sigma^{\prime}\right)} \\
& \times e^{-\Delta^{2} p^{2} / 2-i p \bar{X}\left(\sigma+\sigma^{\prime}\right) / 2}
\end{aligned}
$$

Since the measurement is done after the interaction was completed, namely at a time $t>T$, we set $\bar{X}(t)=\bar{X}=\int_{0}^{T} d t^{\prime} V\left(t^{\prime}\right)$.

The method of solving Eq. (67) with the initial condition (68) is outlined in appendix B. Here we just quote the result, which is

$$
\begin{aligned}
\rho\left(p, K ; \sigma, \sigma^{\prime} ; t\right)= & a_{\sigma^{\prime}}^{*} a_{\sigma} e^{\gamma t} \sqrt{\frac{8 \pi u \Delta^{2}}{2 \Delta^{2}+u}} e^{-\Delta^{2} p^{2} / 2-i p \bar{X}\left(\sigma+\sigma^{\prime}\right) / 2-D \Theta p^{2}} e^{-i \frac{\hbar}{2 M} \lambda K p-u e^{2 \gamma t} K^{2}} \\
& \times \exp \left\{-\frac{\left[\bar{X}\left(\sigma-\sigma^{\prime}\right)+\frac{\hbar}{2 M} \lambda p+i 2 u e^{\gamma t} K\right]^{2}}{4\left(2 \Delta^{2}+u\right)}\right\},
\end{aligned}
$$

where we have defined

$$
\begin{aligned}
u(t) & \equiv 1 / 4 D \eta(t) \\
\lambda(t) & \equiv \frac{\zeta^{2}(t)}{\eta(t)}=\frac{2}{\gamma} \frac{\left(e^{\gamma t}-1\right)}{\left(e^{\gamma t}+1\right)} \\
\Theta(t) & \equiv\left(\frac{\hbar}{M \gamma}\right)^{2}[t-\lambda(t)]
\end{aligned}
$$

Once we have this solution we return to the outcome of a measurement of the position of the pointer. In the present one-dimensional case the probability density to detect the center 
of mass of the pointer around $x$, is determined, as in the previous "free" pointer case by $P(x, t)=\sum_{\sigma, \sigma^{\prime}} \rho\left(x, x, \sigma, \sigma^{\prime} ; t\right)$, namely by the diagonal elements of $\rho$ in real space. We thus have to transform Eq. ([6) back to $\left(x, x^{\prime}\right)$ space and then to set $x=x^{\prime}$ in the resulting density matrix. This can be done conveniently by noting that the Fourier transform from $\left(k, k^{\prime}\right)$ space to $\left(x, x^{\prime}\right)$ space i.e.

$$
\rho\left(x, x^{\prime}, t\right)=\int \frac{d k}{2 \pi} e^{-i k^{\prime} x^{\prime}} \int \frac{d k}{2 \pi} e^{i k x} \rho\left(k, k^{\prime}, t\right)
$$

can be written with new variables, $\theta=\left(x-x^{\prime}\right)$ and $X=\left(x+x^{\prime}\right) / 2$, and the use of the definitions $K=\left(k+k^{\prime}\right) / 2$ and $p=\left(k-k^{\prime}\right)$, as

$$
\rho(X, \theta, t)=\int \frac{d p}{2 \pi} e^{i p X} \int \frac{d K}{2 \pi} e^{i K \theta} \rho(K, p, t) .
$$

Now the probability density will be given simply by $P(x, t)=\sum_{\sigma, \sigma^{\prime}} \rho\left(X=x, \sigma ; \theta=0, \sigma^{\prime} ; t\right)$. We write $P(x, t)$ as a sum of three terms:

$$
P(x, t)=P_{+1}(x, t)+P_{-1}(x, t)+P_{+1,-1}(x, t) .
$$

where $P_{ \pm 1}(x, t)=\rho(x, x, \pm 1, \pm 1 ; t)$ stand for the diagonal elements, and $P_{+1,-1}(x, t)=$ $\rho(x, x,+1,-1 ; t)+\rho(x, x,-1,+1 ; t)$ stands for the off-diagonal elements.

The first and second terms of Eq. (73) are the probabilities of the spin-up and spin-down components of the measured atom. Performing the transformation in Eq. (72) we get for $\sigma^{\prime}=\sigma=+1$

$$
P_{+1}(x, t)=\left|a_{+1}\right|^{2} \sqrt{\frac{1}{2 \pi \Delta_{\beta}^{2}(t)}} \exp \left\{-\frac{(x-\bar{X})^{2}}{2 \Delta_{\beta}^{2}(t)}\right\}
$$

and for $\sigma^{\prime}=\sigma=-1$

$$
P_{-1}(x, t)=\left|a_{-1}\right|^{2} \sqrt{\frac{1}{2 \pi \Delta_{\beta}^{2}(t)}} \exp \left\{-\frac{(x+\bar{X})^{2}}{2 \Delta_{\beta}^{2}(t)}\right\} .
$$

The spatial broadening of the pointer's position, which is due to both the free quantum diffusion and the effect of the bath, is expressed as

$$
\Delta_{\beta}^{2}(t)=\Delta^{2}[\varkappa(t)+\kappa(t)]
$$


where

$$
\varkappa(t)=1+\left(\frac{1}{\gamma \tau_{f}}\right)^{2}\left(1-e^{-\gamma t}\right)^{2},
$$

and

$$
\kappa(t)=\left(\frac{1}{\gamma \tau_{f}}\right)^{2} \frac{4 \Delta^{2} D}{\gamma} f(\gamma t)
$$

is expressed in terms of the function

$$
f(x)=2\left(x-2 \frac{\left(1-e^{-x}\right)}{\left(1+e^{-x}\right)}\right)+\frac{\left(1-e^{-x}\right)^{3}}{\left(1+e^{-x}\right)} .
$$

We now compare these expressions with those obtained previously, Eqs. (26), (27) and (29), in the absence the bath. We observe that the previous broadening of the pointer's position, $\Delta_{f}(t)$, is replaced by $\Delta_{\beta}(t)$, where $\varkappa(t)$ takes care of both the free quantum diffusion and the friction caused by the environment, and $\kappa(t)$ corresponds to the diffusion afflicted on the pointer by the gas surrounding it. The effect of the environment on the probabilities $P_{ \pm 1}(x, t)$ is just to enlarge the uncertainty of the pointer's position.

Now let us look at the off-diagonal term of Eq. (73). Taking $a_{ \pm 1}=\left|a_{ \pm 1}\right| e^{i \varphi_{ \pm}}$we get

$$
\begin{aligned}
P_{+1,-1}(x, t)= & 2 \sqrt{P_{+1}(x, t) P_{-1}(x, t)} \exp \left\{-\frac{\bar{X}^{2} \kappa(t)}{2 \Delta_{\beta}^{2}(t)}\right\} \\
& \times \cos \left(\frac{x \bar{X}}{\Delta_{\beta}^{2}(t) \tau_{f}} \frac{1}{\gamma}\left(1-e^{-\gamma t}\right)+\varphi_{-}-\varphi_{+}\right),
\end{aligned}
$$

where $\kappa(t)$ is given by Eq.(78). This is the main result of the present paper. The function $P_{+1,-1}(x, t)$ represents the probability of finding the system in a state of interference between the two possible "classical" outcomes of the measurement. If we compare this result with the interference term, Eq. (28), of the "free" pointer, we observe two changes: (i) the argument of the cos in Eq.(28) is replaced by a more elaborate time dependent expression in Eq.(80), yet in the limit $\gamma \rightarrow 0$ it reduces to the expression in Eq.(28), and (ii) a new time dependent exponential factor appears in Eq.(80). This exponential factor is the important effect of the environment on the pointer, and we examine it now more closely. Let us write it as $e^{-g(t)}$, where $g(t)$ is the decoherence function, and it is given by 


$$
g(t)=\frac{X_{0}^{2} \kappa(t)}{2 \Delta_{\beta}^{2}(t)}=\frac{\bar{X}^{2}}{2 \Delta^{2}}\left[\frac{\kappa(t)}{\kappa(t)+\varkappa(t)}\right] .
$$

Notice that it is not the friction, which is due to energy exchange with the bath, that is responsible for the decoherence, but rather the diffusion in momentum space, which is originating it.

For times much smaller than the relaxation time, i.e. $\gamma t<<1$, we have

$$
\kappa(t) \rightarrow D\left(\frac{1}{\gamma \tau_{f}}\right)^{2} \frac{\Delta^{2}}{\gamma} \frac{8}{3}(\gamma t)^{3}=2 D\left(\frac{2 \Delta}{\tau_{f}}\right)^{2} \frac{t^{3}}{3},
$$

and since $\varkappa(t)$ is of order 1 ,

$$
g(t) \rightarrow \frac{\bar{X}^{2}}{\Delta^{2}} D\left(\frac{2 \Delta}{\tau_{f}}\right)^{2} \frac{t^{3}}{3} .
$$

Remembering that $D=M \gamma k_{B} T / \hbar^{2}$, we write $g(t)$ as $\left(\Gamma^{\prime} t\right)^{3}$, where

$$
\Gamma^{\prime}=\left(\frac{\bar{X}^{2}}{\Delta^{4}} \frac{\gamma k_{B} T}{3 M}\right)^{1 / 3}=\left[\frac{1}{3} \frac{\bar{X}^{2}}{\Delta^{2}} \frac{\gamma k_{B} T}{\hbar^{2}}\left(\frac{\hbar^{2}}{M \Delta^{2}}\right)\right]^{1 / 3}
$$

plays the role of a kind of inverse decay time of the interference. It is instructive to compare $\Gamma^{\prime}$ of Eq. (84) with the analogous expression of Ref. [6], Eq.(3.5). Their result was obtained for an oscillator, with a typical frequency of $\omega_{R}$, which is coupled to a reservoir of harmonic oscillators, in the high temperature limit, for the weakly damped case, $\gamma / \omega_{R} \ll 1$, and at initial times. The only difference between their $\Gamma_{2}$, and that of our system, $\Gamma^{\prime}$, is that their oscillator's quanta $\hbar \omega_{R}$ is replaced by the energy term $\hbar^{2} / M \Delta^{2}$, which is related to the quantum mechanical energy associated with the initial spread of the pointer. To make a simple estimate for $\Gamma^{\prime}$ let us take our pointer as a silver atom. We thus take $\Delta \sim 1$ $\mu \mathrm{m}$, to represent a pinhole for localizing the atom, $\bar{X} \sim 1 \mathrm{~cm}$, as a deflection position, $M \sim 1.8 \times 10^{-22} \mathrm{~g}$, and $a=1.75 \times 10^{-8} \mathrm{~cm}$. For air at room temperature, namely with $\tau_{r}=1 / \gamma \simeq 0.4 \mathrm{~ns}$, we obtain $\Gamma^{\prime} \tau_{r} \sim 50$. We see that the typical decoherence time $1 / \Gamma^{\prime}$ is almost two orders of magnitude smaller then relaxation time. However this behavior does not proceed for times much longer than the relaxation time. When time increases, so does $g(t)$, and eventually it reaches a saturation value of $g(t) \rightarrow \frac{1}{2}(\bar{X} / \Delta)^{2}$, which is of the order of $10^{8}$. Nevertheless, at later times when $\gamma t>1$, but long before saturation, we have 


$$
\kappa(t) \rightarrow 2 D\left(\frac{2 \Delta}{\gamma \tau_{f}}\right)^{2} t
$$

which gives

$$
g(t) \rightarrow \frac{\bar{X}^{2}}{2 \Delta^{2}}\left[\frac{2 D\left(\frac{2 \Delta}{\gamma_{f}}\right)^{2} t}{2 D\left(\frac{2 \Delta}{\gamma \tau_{f}}\right)^{2} t+1}\right]
$$

At this time range, $g(t)$ is approximately linear in time, i.e., $g(t) \rightarrow \Gamma t$, with a slope given by

$$
\Gamma=\frac{\bar{X}^{2}}{\Delta^{4}} \frac{k_{B} T}{M \gamma}=\frac{\bar{X}^{2}}{\Delta^{2}} \frac{k_{B} T}{\hbar^{2} \gamma} \frac{\hbar^{2}}{M \Delta^{2}},
$$

for the silver atom it yields $\Gamma / \gamma=3.5 \times 10^{5}$. Thus the typical decoherence time $1 / \Gamma$ in the linear part is much shorter then $1 / \Gamma^{\prime}$. The meaning of this result, as was pointed out by Ref. [6], is that as far as the decoherence is concerned, the system becomes aware of the bath, in a time scale much shorter than $\tau_{r}$, and the interference is deteriorating almost immediately. Our decoherence rate, $\Gamma$, of Eq. (87), for the gas bath, is quite similar to that of the oscillators' bath of Ref. [6], Eq.(3.7), at the high temperature and strongly damped limit. It is interesting to note that the strongly damped limit, which is defined as $R_{\text {osc }}=\gamma / \omega_{R} \gg 1$ for the oscillators' bath, is replaced here by $R_{f}=\gamma \tau_{f} \gg 1$. In our analysis the meaning of the "strongly damped" case is not clear, yet taking $\hbar^{2} / M \Delta^{2}$ instead of the oscillator's quanta $\hbar \omega_{R}$ of Ref. [6], we see that $R_{f}=\gamma \tau_{f} \sim 10^{7}$ is indeed very large. So in that sense we are in the strongly damped limit. It is also interesting to compare $\Gamma$ of Eq. (87) with the decoherence rate, $\Gamma_{Z} \simeq \gamma\left(\bar{X} / \lambda_{T}\right)^{2}$, quoted by Zurek Ref. [2], where $\lambda_{T}=\hbar / \sqrt{2 M k_{B} T}$ is the thermal de Broglie wave length of the pointer. Apparently Zurek assumes that the decoherence time, is proportional to the inverse of the coefficient of the last term in his equation (9). We find that the ratio $\Gamma / \Gamma_{Z}$ is of order $1 / R_{f}^{2}$, i.e., the decoherence time due to the interaction of the pointer with the gas is much longer than Zurek estimated. This is also the case for the Caldeira and Leggett [6] decoherence rates, $\Gamma_{1}$ and $\Gamma_{3}$ : The ratio $\Gamma_{1} / \Gamma_{Z}$ in the weakly damped limit, is of order $R_{\text {ocs }} / R_{f}$, while in the strongly damped limit, $\Gamma_{3} / \Gamma_{Z}$ is of order $1 / R_{f} R_{o s c}$. 
Fig. 1 shows a graphical description summarizing the discussion above. The decoherence

function, $g(t)$ of Eq. (81), normalized to the saturation value $\frac{1}{2}(\bar{X} / \Delta)^{2}$ is depicted as a function of $\gamma t$. In the inset, we see more closely the behavior at initial times, where $g(t)$ starts as $\left(\Gamma^{\prime} t\right)^{3}$ and quickly goes into the linear behavior as $\Gamma t$.

\section{CONCLUSIONS}

In this paper we have studied the affect of the environment on the process of measurement of the state of a microscopic spin particle by a classical pointer. It has been shown that, the entangled state, which occurred due to the interaction of the spin particle with the measuring apparatus, the pointer, is developed into a mixed state by the environment, in an extremely short time. An initially prepared state of a spin half particle, as a superposition of up and down states, is transformed by the interaction into corresponding two distinguishable positions of the pointer. The probability to find the pointer in these positions is expressed in terms of two separated wave-packets, and an interference term, which is a manifestation of the entanglement between the particle and the pointer. Due to the coupling of the pointer with the environment, which is modeled here as a gas of independent particles, rather than an ensemble of harmonic oscillators, this quantum interference decays extremely fast in time. The final mixed state, which is the outcome of the decoherence, introduced by the environment, is being expressed as the two separated wave-packets of the pointer's position. The probabilistic nature of quantum mechanics is still maintained in the final representation of the pointer, but the interference is destroyed. An exponential decay function, which portrays the decoherence, is expressed in terms of the parameters of the pointer and the bath.

\section{APPENDIX A}




\section{A. Solving the Fokker-Planck Equation for the Pointer}

In the present Appendix we seek the solution for Eq. (67) with the initial condition (68). It will be convenient to study the density matrix as a Wigner distribution function, which is defined by the transformation

$$
\rho(K, p, t)=\int_{-\infty}^{\infty} d X e^{-i X p} W(X, K, t) .
$$

Eq. (67) is transformed to the equation

$$
\begin{aligned}
\frac{\partial}{\partial t} W(X, K, t)+ & \frac{\hbar K}{M} \frac{\partial}{\partial X} W(X, K, t) \\
& =\gamma \frac{\partial}{\partial K}(K W(X, K, t))+D \frac{\partial^{2}}{\partial K^{2}} W(X, K, t)
\end{aligned}
$$

where $W(X, K, t)$ plays a role analogous to that of a classical distribution function, $f(x, p, t)$ to find a massive test particle near the position $x$ and the momentum $p$, while moving in

a gaseous bath. This partial differential equation can be solved by the use of the method of characteristics. We start by ignoring the term with the second order derivative in $K$, and we look for curves that satisfy the conditions

$$
\frac{d X}{\hbar K / M}=d t=-\frac{d K}{\gamma K}=\frac{d W}{\gamma W} .
$$

Integrating with respect to $t$ we have

$$
\begin{gathered}
d t=-\frac{d K}{\gamma K} \rightarrow K=\kappa e^{-\gamma t} \rightarrow \kappa=K e^{\gamma t} \\
\frac{d X}{\hbar K / M}=d t \rightarrow X=-\frac{\hbar}{\gamma M} \kappa e^{-\gamma t}+\xi \rightarrow \xi=X+\frac{\hbar K}{M \gamma} \\
d t=\frac{d W}{\gamma W} \rightarrow W=\omega e^{\gamma t}
\end{gathered}
$$

where we wrote the constants of integration over $t$, i.e. $\kappa, \xi$ and $\omega$, in terms of the variables $K, X, t$.

We can now make the independent variables' transformation $K, X, t \rightarrow \kappa, \xi, \tau$, where 


$$
\begin{aligned}
& \kappa=K e^{\gamma t} \\
& \xi=X+\frac{\hbar K}{M \gamma} \\
& \tau=t
\end{aligned}
$$

and find that in terms of the new variables, and the substitution

$$
W(\xi, \kappa, \tau)=\omega(\xi, \kappa, \tau) e^{\gamma \tau}
$$

Eq. (89) takes the form

$$
\frac{\partial}{\partial \tau} \omega(\xi, \kappa, \tau)=D\left(e^{\gamma \tau} \frac{\partial}{\partial \kappa}+\frac{\hbar}{M \gamma} \frac{\partial}{\partial \xi}\right)^{2} \omega(\xi, \kappa, \tau)
$$

This equation is a diffusion equation in $\xi, \kappa, \tau$ space, with a time dependent diffusion coefficient. This can be solved for example by using Fourier transform in $\xi, \kappa$ space, namely, introducing $f(p, x, \tau)$ by

$$
f(p, x, \tau)=\frac{1}{2 \pi} \int_{-\infty}^{\infty} d \xi e^{-i \xi p} \int_{-\infty}^{\infty} d x e^{i \kappa x} \omega(\xi, \kappa, \tau)
$$

Eq. (96) is simply replaced by

$$
\frac{\partial}{\partial \tau} f(p, x, \tau)=-D\left(e^{\gamma \tau} x-\frac{\hbar}{M \gamma} p\right)^{2} f(p, x, \tau)
$$

with the formal solution

$$
f(p, x, \tau)=f(p, x, 0) \exp \left\{-D \int_{0}^{\tau} d \tau^{\prime}\left(e^{\gamma \tau^{\prime}} x-\frac{\hbar}{M \gamma} p\right)^{2}\right\}
$$

where $f(p, x, 0)$ is the initial value of $f$. If we denote by

$$
\begin{aligned}
& \eta(t)=\int_{0}^{t} d t^{\prime} e^{2 \gamma t^{\prime}}=\frac{e^{2 \gamma t}-1}{2 \gamma} \\
& \zeta(t)=\int_{0}^{t} d t^{\prime} e^{\gamma t^{\prime}}=\frac{e^{\gamma t}-1}{\gamma},
\end{aligned}
$$

we can write Eq. (99) as

$$
f(p, x, \tau)=f(p, x, 0) \exp \left\{-D\left(\eta(\tau) x^{2}-2 \zeta(\tau) \frac{\hbar}{M \gamma} x p+\left(\frac{\hbar}{M \gamma}\right)^{2} \tau p^{2}\right)\right\} .
$$


We return now to Eq. (97) to find

$$
\begin{aligned}
\omega(\xi, \kappa, \tau)= & \frac{1}{2 \pi} \int_{-\infty}^{\infty} d p e^{i p \xi} \int_{-\infty}^{\infty} d x e^{-i x \kappa} \\
& e^{-D\left(\eta(\tau) x^{2}-2 \zeta(\tau) \frac{\hbar}{M \gamma} x p+\left(\frac{\hbar}{M \gamma}\right)^{2} \tau p^{2}\right)} f(p, x, 0) \\
= & \frac{1}{(2 \pi)^{2}} \int_{-\infty}^{\infty} d p e^{i p \xi} \int_{-\infty}^{\infty} d x e^{-i x \kappa} \int_{-\infty}^{\infty} d \xi^{\prime} e^{-i \xi^{\prime} p} \int_{-\infty}^{\infty} d \kappa^{\prime} e^{i \kappa^{\prime} x} \\
& e^{-D\left(\eta(\tau) x^{2}-2 \zeta(\tau) \frac{\hbar}{M \gamma} x p+\left(\frac{\hbar}{M \gamma}\right)^{2} \tau p^{2}\right)} \omega\left(\xi^{\prime}, \kappa^{\prime}, 0\right) .
\end{aligned}
$$

Note that at $t=0$ we have $W(\xi, \kappa, 0)=\omega(\xi, \kappa, 0)$ and the transformation in Eq. (94) has the form

$$
\begin{aligned}
& \kappa^{\prime}=K^{\prime} \\
& \xi^{\prime}=X^{\prime}+\frac{\hbar K^{\prime}}{M \gamma},
\end{aligned}
$$

hence we can write Eq. (102) in terms of the Wigner distribution

$$
\begin{aligned}
& W(\xi, \kappa, \tau)=e^{\gamma \tau-i \kappa\left(\sigma-\sigma^{\prime}\right) X_{0}} \frac{1}{2 \pi} \int_{-\infty}^{\infty} d p e^{i p \xi} \int_{-\infty}^{\infty} d x e^{-i x \kappa} \\
& e^{-D\left(\eta(\tau) x^{2}-2 \zeta(\tau) \frac{\hbar}{M \gamma} x p+\left(\frac{\hbar}{M \gamma}\right)^{2} \tau p^{2}+\left(\sigma-\sigma^{\prime}\right) X_{0}\left[\eta(\tau)\left(\sigma-\sigma^{\prime}\right) X_{0}-2 \zeta(\tau) \frac{\hbar}{M \gamma} p+2 \eta(\tau) x\right]\right)} f(p, x, 0) \\
& =\frac{1}{(2 \pi)^{2}} e^{\gamma t-i K e^{\gamma t}\left(\sigma-\sigma^{\prime}\right) X_{0}} \int_{-\infty}^{\infty} d p e^{i p\left(X+\frac{\hbar K}{M \gamma}\right)} \int_{-\infty}^{\infty} d x \\
& \times e^{-i x K e^{\gamma t}} e^{-D\left(\eta(t) x^{2}-2 \zeta(t) \frac{\hbar}{M \gamma} x p+\left(\frac{\hbar}{M \gamma}\right)^{2} t p^{2}+\left(\sigma-\sigma^{\prime}\right) X_{0}\left[\eta(t)\left(\sigma-\sigma^{\prime}\right) X_{0}-2 \zeta(t) \frac{\hbar}{M \gamma} p+2 \eta(t) x\right]\right)} \\
& \int_{-\infty}^{\infty} d X^{\prime} e^{-i\left(X^{\prime}+\frac{\hbar K^{\prime}}{M \gamma}\right) p} \int_{-\infty}^{\infty} d K^{\prime} e^{i K^{\prime} x} W\left(X^{\prime}, K^{\prime}, 0\right) .
\end{aligned}
$$

Using Eq. (94) and Eq. (95) the Wigner distribution at time $t$ can be then expressed in terms of its initial value as

$$
W(X, K, t)=\frac{1}{2 \pi} \int_{-\infty}^{\infty} d X^{\prime} \int_{-\infty}^{\infty} d K^{\prime} J\left(X, K, t ; X^{\prime}, K^{\prime}, 0\right) W\left(X^{\prime}, K^{\prime}, 0\right),
$$

where $J$ is the propagator function for the Wigner distribution,

$$
\begin{aligned}
J\left(X, K, t ; X^{\prime}, K^{\prime}, 0\right)= & e^{\gamma t} \frac{1}{2 \pi} \int_{-\infty}^{\infty} d p e^{i p\left[\left(X-X^{\prime}\right)+\frac{\hbar}{M \gamma}\left(K-K^{\prime}\right)\right]} \int_{-\infty}^{\infty} d x e^{-i x\left(e^{\gamma t} K-K^{\prime}\right)} \\
& e^{-D\left(\eta(t) x^{2}-2 \zeta(t) \frac{\hbar}{M \gamma} x p+\left(\frac{\hbar}{M \gamma}\right)^{2} t p^{2}\right)}
\end{aligned}
$$

We can write this propagator explicitly by preforming the integrations over $x$ and $p$, which gives 


$$
J\left(X, K, t ; X^{\prime}, K^{\prime}, 0\right)=e^{\gamma t} \sqrt{\frac{u}{D \Theta}} e^{-u\left(e^{\gamma t} K-K^{\prime}\right)^{2}} e^{-\Phi / 4 D \Theta},
$$

where we used the following definitions

$$
\begin{aligned}
u(t) & \equiv 1 / 4 D \eta(t) \\
\lambda(t) & \equiv \frac{\zeta^{2}(t)}{\eta(t)}=\frac{2}{\gamma} \frac{\left(e^{\gamma t}-1\right)}{\left(e^{\gamma t}+1\right)}, \\
\vartheta\left(K, K^{\prime}, t\right) & \equiv \frac{\hbar}{2 M} \lambda(t)\left(K+K^{\prime}\right), \\
\Phi\left(X, K, X^{\prime}, K^{\prime}, t\right) & \equiv\left[\left(X-X^{\prime}\right)-\vartheta\right]^{2}, \\
\Theta(t) & \equiv\left(\frac{\hbar}{M \gamma}\right)^{2}[t-\lambda(t)] .
\end{aligned}
$$

Since we are interested in the density matrix in $(K, p)$, we proceed by transforming back the Wigner distribution in Eq. (104) using the inverse of Eq. (88), and write

$$
\begin{aligned}
\rho\left(p, K ; \sigma, \sigma^{\prime} ; t\right) & =\frac{1}{2 \pi} \int_{-\infty}^{\infty} d X^{\prime} \int_{-\infty}^{\infty} d K^{\prime} W\left(X^{\prime}, K^{\prime} ; \sigma, \sigma^{\prime} ; 0\right) \int_{-\infty}^{\infty} d X e^{-i X p} J\left(X, K, t ; X^{\prime}, K^{\prime}, 0\right) \\
& =\frac{1}{2 \pi} \int_{-\infty}^{\infty} d K^{\prime} \int_{-\infty}^{\infty} d X^{\prime} \widetilde{J}\left(p, K, t ; X^{\prime}, K^{\prime}, 0\right) W\left(X^{\prime}, K^{\prime} ; \sigma, \sigma^{\prime} ; 0\right),
\end{aligned}
$$

where the transform of the propagator is given explicitly, with the help of Eq. (106), by

$$
\begin{aligned}
\widetilde{J}\left(p, K, t ; X^{\prime}, K^{\prime}, 0\right) & =\int_{-\infty}^{\infty} d X e^{-i X p} J\left(X, K, t ; X^{\prime}, K^{\prime}, 0\right) \\
& =e^{\gamma t} \sqrt{4 \pi u} e^{-u\left(e^{\gamma t} K-K^{\prime}\right)^{2}} e^{-i X^{\prime} p} e^{-D \Theta p^{2}-i \vartheta p} .
\end{aligned}
$$

Looking at the integral over $X^{\prime}$ in Eq. (108), we note that the $X^{\prime}$ dependence of the propagator $\widetilde{J}\left(p, K, t ; X^{\prime}, K^{\prime}, 0\right)$ as it appears in Eq. (109), is only through the factor $e^{-i X^{\prime} p}$. Hence this integral is actually a Furrier transform of the initial Wigner function, which bring us back to the initial density matrix

$$
\rho\left(p, K ; \sigma, \sigma^{\prime} ; 0\right)=\int_{-\infty}^{\infty} d X^{\prime} e^{-i X^{\prime} p} W\left(X^{\prime}, K^{\prime} ; \sigma, \sigma^{\prime} ; 0\right) .
$$

This last result enable us to write the density matrix at any time $t$, in terms of the density matrix at time $t=0$

$$
\rho\left(p, K ; \sigma, \sigma^{\prime} ; t\right)=\frac{1}{2 \pi} \int_{-\infty}^{\infty} d K^{\prime} \widetilde{J}_{\rho}\left(p, K, t ; K^{\prime}, 0\right) \rho\left(p, K^{\prime} ; \sigma, \sigma^{\prime} ; 0\right)
$$


where here the propagator is given simply by Eq. (109) without the $e^{-i X^{\prime} p}$ factor

$$
\widetilde{J}_{\rho}\left(p, K, t ; K^{\prime}, 0\right)=e^{\gamma t} \sqrt{4 \pi u} e^{-u\left(e^{\gamma t} K-K^{\prime}\right)^{2}} e^{-D \Theta p^{2}-i \vartheta p} .
$$

Consider now the initial condition for the density matrix (68)

$$
\rho\left(p, K ; \sigma, \sigma^{\prime}, 0\right)=a_{\sigma^{\prime}}^{*} a_{\sigma} 2\left(2 \pi \Delta^{2}\right)^{1 / 2} e^{-2 \Delta^{2} K^{2}-i K X_{0}\left(\sigma-\sigma^{\prime}\right)} e^{-\Delta^{2} p^{2} / 2-i p X_{0}\left(\sigma+\sigma^{\prime}\right) / 2} .
$$

Substituting Eq. (113) into Eq. (111), using Eq. (112) and preforming the integration over $K^{\prime}$, we get the explicit form of the density matrix in $(p, K)$ space, at time $t$,

$$
\begin{aligned}
\rho\left(p, K ; \sigma, \sigma^{\prime} ; t\right)= & a_{\sigma^{\prime}}^{*} a_{\sigma} e^{\gamma t} \sqrt{\frac{8 \pi u \Delta^{2}}{2 \Delta^{2}+u}} e^{-\Delta^{2} p^{2} / 2-i p X_{0}\left(\sigma+\sigma^{\prime}\right) / 2-D \Theta p^{2}} e^{-i \frac{\hbar}{2 M} \lambda K p-u e^{2 \gamma t} K^{2}} \\
& \times \exp \left\{-\frac{\left[X_{0}\left(\sigma-\sigma^{\prime}\right)+\frac{\hbar}{2 M} \lambda p+i 2 u e^{\gamma t} K\right]^{2}}{4\left(2 \Delta^{2}+u\right)}\right\},
\end{aligned}
$$

which is our goal in this derivation.

\section{APPENDIX B}

\section{A. The Pointer in a Random Velocity Field}

In Section IV we derived the master equation for the behavior of the pointer under the influence of the reservoir. Our starting point was the explicit form of the interaction Hamiltonian $\phi$, which after the averaging over the bath determined the inverse relaxation time $\gamma$. As a complement to this explicit derivation, it will be instructive to look at a much simpler model, in which the effect of the environment is described in terms of a random velocity field, $\mathbf{v}(t)$. The interaction of the pointer with the bath, which is simulated by this random

field, perturbs the meter and introduces decoherence. Namely it destroys the interference by introducing randomization of the phases, and making the pointer's readings distinguishable. The development in time of the entire system is described by unitary transformation, which originates probability into the dynamics by quantum mechanics. This simple model is interesting because in addition to being readily understood, it gives essentially the same behavior as the more elaborate treatment of section IV. 
Thus taking the Hamiltonian (9) and adding the random field interaction we have

$$
H=\frac{\mathbf{P}^{2}}{2 M}+V(t) P_{x} \sigma_{z}+\mathbf{v}(t) \cdot \mathbf{P} .
$$

The effect of the environment on the pointer is assumed to be represented by the interaction Hamiltonian

$$
H_{P B}=\mathbf{v}(t) \cdot \mathbf{P} .
$$

Here $\mathbf{v}(t)$ is a random vector field, which simulates the random impacts suffered by the center of mass of the pointer, while in contact with the bath around it. The reservoir itself can be thought of as an ensemble of many external particles, which collide with the pointer. The choice of this Hamiltonian can be thought of as an extension of the spin particle Hamiltonian, $H_{S P}$. Namely, it is viewed as corresponding to many individual impacts on the pointer momentum $\mathbf{P}$, much like the impact on the center of mass, due to the atomic system, Eq. (3). In our simple model the reservoir is not represented by a Hamiltonian, but rather by the characterization of the random "velocity" field, to be introduced at the proper stage of the analysis.

This turns Eq. (10) into

$$
\begin{aligned}
i \hbar \dot{\Psi}(\mathbf{R}, \sigma, t)= & \left(-\frac{\hbar^{2}}{2 M} \frac{\partial^{2}}{\partial \mathbf{R}^{2}}-i \hbar V(t) \sigma \frac{\partial}{\partial X}\right. \\
& \left.-i \hbar \mathbf{v}(t) \cdot \frac{\partial}{\partial \mathbf{R}}\right) \Psi(\mathbf{R}, \sigma, t),
\end{aligned}
$$

and Eq. (13) into

$$
i \hbar \dot{\Psi}(\mathbf{k}, \sigma, t)=\left(\frac{\hbar^{2} k^{2}}{2 M}+\hbar V(t) k_{x} \sigma+\hbar \mathbf{k} \cdot \mathbf{v}(t)\right) \Psi(\mathbf{k}, \sigma, t) .
$$

The solution of this equation is then

$$
\Psi(\mathbf{k}, \sigma, t)=\Psi(\mathbf{k}, \sigma, 0) e^{-i \omega_{k} t-i \bar{X}(t) k_{x} \sigma-i \mathbf{k} \cdot \mathbf{x}(\mathbf{t})},
$$

where

$$
\mathbf{x}(t)=\int_{0}^{t} d t^{\prime} \mathbf{v}\left(t^{\prime}\right)
$$


is also a random field with the dimensions of length, and the density matrix is

$$
\begin{aligned}
\rho\left(\mathbf{k}, \sigma ; \mathbf{k}^{\prime}, \sigma^{\prime} ; t\right)= & \rho\left(\mathbf{k}, \sigma ; \mathbf{k}^{\prime}, \sigma^{\prime} ; 0\right) e^{-i\left(\omega_{k}-\omega_{k^{\prime}}\right) t} \\
& \times e^{-i \bar{X}(t)\left(k_{x} \sigma-k_{x}^{\prime} \sigma^{\prime}\right)-i\left(\mathbf{k}-\mathbf{k}^{\prime}\right) \cdot \mathbf{x}(t)} .
\end{aligned}
$$

Since $\mathbf{x}(t)$ is a random field it should be expressed in terms of a distribution, $W[\mathbf{x}(t)]$. The velocity field is a sum of many "impulses", so does the length random field, $\mathbf{x}(t)$, namely

$$
\begin{aligned}
& \mathbf{v}(t)=\sum_{i} \mathbf{v}_{i}(t)=\sum_{i} \mathbf{v}_{i} f\left(t-t_{i}\right) \\
& \mathbf{x}(t)=\sum_{i} \mathbf{x}_{i}=\sum_{i} \mathbf{v}_{i} \tau_{i} .
\end{aligned}
$$

Here $\mathbf{v}_{i}$ is the "strength" of the $i$-th velocity impulse, $f\left(t-t_{i}\right)$ samples the $i$-th impulse, whose duration is $\tau_{i}$, and the summation is carried out over all the impulses which occurred during the integration time $t$ in Eq. (120). Assume that the probability density of the individual $\mathbf{x}_{i}$ is Gaussian, namely that

$$
P\left(\mathbf{x}_{i}\right)=\left(2 \pi \sigma_{i}^{2}\right)^{-3 / 2} \exp \left(-\mathbf{x}_{i}^{2} / 2 \sigma_{i}^{2}\right) .
$$

The single parameter, $\sigma_{i}$, was introduced to represent the width of the $\mathbf{x}_{i}$ distribution, which is also assumed to be spherically symmetric. The probability density to find $\mathbf{x}(t)$, at the time $t$, to be between $\mathbf{x}$ and $\mathbf{x}+d \mathbf{x}$ is then

$$
W[\mathbf{x}(t)]=\prod_{i} \int d \mathbf{x}_{i} P\left(\mathbf{x}_{i}\right) \delta\left[\mathbf{x}(t)-\sum_{j} \mathbf{x}_{j}\right] .
$$

We now transform the $\delta$-function as

$$
\delta\left[\mathbf{x}(t)-\sum_{j} \mathbf{x}_{j}\right]=\frac{1}{(2 \pi)^{3}} \int_{-\infty}^{+\infty} d \mathbf{q} e^{-i \mathbf{q} \cdot\left[\mathbf{x}(t)-\sum_{j} \mathbf{x}_{j}\right]}
$$

and Eq. (124) is written as

$$
\begin{aligned}
W[\mathbf{x}(t)]= & \frac{1}{(2 \pi)^{3}} \int_{-\infty}^{+\infty} d \mathbf{q} e^{-i \mathbf{q} \cdot \mathbf{x}(t)} \\
& \times \prod_{i} \int d \mathbf{x}_{i} P\left(\mathbf{x}_{i}\right) e^{i \mathbf{q} \cdot \mathbf{x}_{i}}
\end{aligned}
$$


Performing the $\mathbf{x}_{i}$-integration using Eq. (123),

$$
\int_{-\infty}^{+\infty} d \mathbf{x}_{i} P\left(\mathbf{x}_{i}\right) e^{i \mathbf{q} \cdot \mathbf{x}_{i}}=e^{-\sigma_{i}^{2} q^{2} / 2}
$$

the product of the exponentials yields

$$
W[\mathbf{x}(t)]=\frac{1}{(2 \pi)^{3}} \int_{-\infty}^{+\infty} d \mathbf{q} e^{-i \mathbf{q} \cdot \mathbf{x}(t)} e^{-(1 / 2) q^{2} \sum_{i} \sigma_{i}^{2}}
$$

Given that the impulses' rate, i.e., the number of impulses per unit time is $\nu$, and that the average width of the $\sigma_{i}$-distribution is $\bar{\sigma}$, we get

$$
\sum_{i} \sigma_{i}^{2} \rightarrow \nu \bar{\sigma}^{2} t
$$

and finally,

$$
\begin{aligned}
W[\mathbf{x}(t)] & =\frac{1}{(2 \pi)^{3}} \int_{-\infty}^{+\infty} d \mathbf{q} e^{-i \mathbf{q} \cdot \mathbf{x}(t)} e^{-(1 / 2) q^{2} \nu \bar{\sigma}^{2} t} \\
& =\frac{1}{\left(2 \pi \nu \bar{\sigma}^{2} t\right)^{3 / 2}} \exp \left(-\frac{\mathbf{x}^{2}(t)}{2 \nu \bar{\sigma}^{2} t}\right)
\end{aligned}
$$

Once the Distribution of $\mathbf{x}(t)$ is known, we can find the average of the density matrix in k-space. Notice that we could first return to the real space by Eq. (12), and then perform the averaging. The average over the random field is simply

$$
\int_{-\infty}^{+\infty} d \mathbf{x} W(\mathbf{x}) e^{-i\left(\mathbf{k}-\mathbf{k}^{\prime}\right) \cdot \mathbf{x}}=e^{-\left(\beta^{2}\left(\mathbf{k}-\mathbf{k}^{\prime}\right)^{2} / 2\right)} .
$$

where

$$
\beta^{2}=\nu \bar{\sigma}^{2} t
$$

Notice that Eq. (131) is similar to the characteristic function of the distribution in $\mathbf{x}$.

The density matrix of Eq.(121), averaged over the distribution function of Eq.(130), and denoted by $\bar{\rho}$, is

$$
\begin{aligned}
\bar{\rho}\left(\mathbf{k}, \sigma ; \mathbf{k}^{\prime}, \sigma^{\prime} ; t\right)= & \rho\left(\mathbf{k}, \sigma ; \mathbf{k}^{\prime}, \sigma^{\prime} ; 0\right) e^{-i\left(\omega_{k}-\omega_{k^{\prime}}\right) t} \\
& \times e^{-i \bar{X}\left(k_{x} \sigma-k_{x}^{\prime} \sigma^{\prime}\right)-\beta^{2}\left(\mathbf{k}-\mathbf{k}^{\prime}\right)^{2} / 2}
\end{aligned}
$$


We now use Eq. (18) and Eq. (12) to return to real space and find

$$
\begin{aligned}
\bar{\rho}\left(\mathbf{R}, \sigma ; \mathbf{R}^{\prime}, \sigma^{\prime} ; t\right)= & a_{\sigma^{\prime}}^{*} a_{\sigma}\left(8 \pi \Delta^{2}\right)^{3 / 2} \frac{1}{(2 \pi)^{6}} \times \\
& \int_{-\infty}^{+\infty} d^{3} k^{\prime} e^{-i \mathbf{k}^{\prime} \cdot \mathbf{R}^{\prime}} \int_{-\infty}^{+\infty} d^{3} k e^{i \mathbf{k} \cdot \mathbf{R}} e^{-\Delta^{2}\left(\mathbf{k}^{2}+\mathbf{k}^{\prime 2}\right)} e^{-i\left(\omega_{k}-\omega_{k^{\prime}}\right) t-i \overline{\mathbf{R}} \cdot\left(\mathbf{k} \sigma-\mathbf{k}^{\prime} \sigma^{\prime}\right)-\beta^{2}\left(\mathbf{k}-\mathbf{k}^{\prime}\right)^{2} / 2}
\end{aligned}
$$

where, for convenience of calculation, we have introduced a deflection vector

$$
\overline{\mathbf{R}}=(\bar{X}, 0,0)
$$

We notice that the integral in Eq. (134) is a product of three equivalent integrals,

$$
\bar{\rho}\left(\mathbf{R}, \sigma ; \mathbf{R}^{\prime}, \sigma^{\prime} ; t\right)=a_{\sigma^{\prime}}^{*} a_{\sigma} I_{x} I_{y} I_{z}
$$

where, for $i=x, y, z$,

$$
\begin{aligned}
I_{i} & =\left(8 \pi \Delta^{2}\right)^{1 / 2} \frac{1}{(2 \pi)^{2}} \int_{-\infty}^{+\infty} d k_{i}^{\prime} e^{-i k_{i}^{\prime} R_{i}^{\prime}} \int_{-\infty}^{+\infty} d k_{i} e^{i k_{i} R_{i}} e^{-\Delta^{2}\left(k_{i}^{2}+k_{i}^{\prime 2}\right)} e^{-i\left(\omega_{k_{i}}-\omega_{k_{i}^{\prime}}\right) t-i \bar{R}_{i}\left(k_{i} \sigma-k_{i}^{\prime} \sigma^{\prime}\right)-\beta^{2}\left(k_{i}-k_{i}^{\prime}\right)^{2} / 2} \\
& =\left(\frac{1}{2 \pi \Delta_{\beta}^{2}(t)}\right)^{1 / 2} \exp \left[-\frac{1}{4 \Delta_{\beta}^{2}(t)}\left(\zeta(t) \Omega_{i}^{\prime 2}+\zeta^{*}(t) \Omega_{i}^{2}+\left(\Omega_{i}^{\prime}-\Omega_{i}\right)^{2}\left(\beta^{2} / 2 \Delta^{2}\right)\right)\right]
\end{aligned}
$$

Here we have introduced

$$
\begin{aligned}
\Omega_{i} & =R_{i}-\bar{R}_{i} \sigma, \\
\Omega_{i}^{\prime} & =R_{i}^{\prime}-\bar{R}_{i} \sigma^{\prime} \\
\Delta_{\beta}^{2}(t) & =\Delta^{2}\left[\xi(t)+\left(\beta^{2} / \Delta^{2}\right)\right],
\end{aligned}
$$

where in the last equation, $\Delta_{\beta}^{2}(t)$ reflects the spatial broadening of the pointer's position. This is due to both the free quantum diffusion, and an additional diffusion term, $\beta^{2}$, caused by the random field $\mathbf{v}(t)$, which simulates the environment. Using Eq. (137) we can write the three dimensional density matrix of Eq. (134) as

$$
\begin{aligned}
\bar{\rho}\left(\mathbf{R}, \sigma ; \mathbf{R}^{\prime}, \sigma^{\prime} ; t\right)= & a_{\sigma^{\prime}}^{*} a_{\sigma}\left(\frac{1}{2 \pi \Delta_{\beta}^{2}(t)}\right)^{3 / 2} \\
& \times \exp \left[-\frac{1}{4 \Delta_{\beta}^{2}(t)}\left(\zeta(t) \Omega^{\prime 2}+\zeta^{*}(t) \Omega^{2}+\left(\Omega^{\prime}-\Omega\right)^{2} \frac{\beta^{2}}{2 \Delta^{2}}\right)\right],
\end{aligned}
$$


where we now have

$$
\begin{aligned}
\Omega & \equiv \mathbf{R}-\widehat{\mathbf{x}} \bar{X} \sigma \\
\Omega^{\prime} & \equiv \mathbf{R}^{\prime}-\widehat{\mathbf{x}} \bar{X} \sigma^{\prime} .
\end{aligned}
$$

Eq. (139) is our result for the reduced density matrix of the pointer in space, after the environment was "traced out". Notice that the spin indices can be considered as parameters for the pointer's position. It is clearly seen that when $\beta \rightarrow 0$ Eq. (139) is reduced back to Eq. (19).

We now turn to calculate the probability to locate the pointer in the position $\mathbf{R}$ in space. We set $\mathbf{R}^{\prime}=\mathbf{R}$ in Eq.(139), and find the diagonal elements in space of the density matrix, $\bar{\rho}\left(\mathbf{R}, \sigma ; \mathbf{R}, \sigma^{\prime} ; t\right)$. Again we write this spatial probability density, as in Eq. (25), as a sum of three terms. The first term is correlated with the spin up state, and we set $\sigma^{\prime}=\sigma=+1$, or,

$$
\Omega^{\prime}=\Omega=\mathbf{R}-\hat{\mathbf{x}} \bar{X},
$$

which leads to:

$$
\begin{aligned}
P_{+1}(\mathbf{R}, t)= & \left|a_{+1}\right|^{2}\left(\frac{1}{2 \pi \Delta_{\beta}^{2}(t)}\right)^{3 / 2} \\
& \times \exp \left(-\frac{1}{2 \Delta_{\beta}^{2}(t)}[\mathbf{R}-\hat{\mathbf{x}} \bar{X}]^{2}\right) .
\end{aligned}
$$

The second term is correlated with the spin down state, and we set $\sigma^{\prime}=\sigma=-1$, or,

$$
\Omega^{\prime}=\Omega=\mathbf{R}+\hat{\mathbf{x}} \bar{X},
$$

and find

$$
\begin{aligned}
P_{-1}(\mathbf{R}, t)= & \left|a_{-1}\right|^{2}\left(\frac{1}{2 \pi \Delta_{\beta}^{2}(t)}\right)^{3 / 2} \\
& \times \exp \left(-\frac{1}{2 \Delta_{\beta}^{2}(t)}[\mathbf{R}+\hat{\mathbf{x}} \bar{X}]^{2}\right) .
\end{aligned}
$$

The probabilities of Eqs. (142) and (144) are similar to these of Eqs. (26) and (27). The only difference is that the free diffusion spread of the Gaussians, $\Delta_{f}^{2}(t)$, is replaced by $\Delta_{\beta}^{2}(t)$ 
of Eq. (138), which adds the effect of the environment on the pointer's position. We thus observe that the probability to find the pointer, either deflected by $+\bar{X}$ for the spin up case, or by $-\bar{X}$ for the spin down case, is "performing" a diffusion-like motion in space, which is strongly affected by the random impulses inflicted by the environment. It is interesting to notice that even in the absence of interaction with the spin particle (i.e. if $\bar{X}=0$ ), the pointer's position would have shown diffusion-like motion of its Gaussian wave-packet, with the same time dependent spread, $\Delta_{\beta}^{2}(t)$.

This diffusion-like behavior of the pointer, due to the interaction with the environment, is a reminiscent of the classical diffusion of a Brownian particle immersed in a fluid [19]. If we compare the result of Eq. (130) with the analogous one of the Brownian motion, we find that the diffusion coefficient is $D=\beta^{2} /(2 t)$ or with Eq. (132) $D=\nu \bar{\sigma}^{2} / 2$. This can provide us with an estimate for $\bar{\sigma}$. Assuming that the environment is a bath in thermal equilibrium with temperature $T$, then, in analogy with Brownian particle, the classical limit yields $\bar{\sigma}^{2} \rightarrow 2 k_{B} T /\left(M \nu^{2}\right)$, where $k_{B}$ is Boltzmann's constant. If we consider the environment as an ensemble of harmonic oscillators, with a typical frequency of $\omega_{0}$, we get

$$
\bar{\sigma}^{2} \rightarrow\left(\frac{2}{M \nu^{2}}\right) \frac{\hbar \omega_{0}}{e^{\left(\hbar \omega_{0} / k_{B} T\right)}-1},
$$

which approaches the classical limit when $\left(\hbar \omega_{0} / k_{B} T\right) \ll 1$. The collision rate, $\nu$, which is the inverse relaxation time, $\tau_{r}$, of the pointer, depends of course on the character of the bath.

It is also instructive to estimate the time scale, on which this diffusion broadening becomes so wide, as to erase the record of the measurement. We can estimate this time, $t_{\text {bluer }}$, by noting that when the width $\Delta_{\beta}\left(t_{\text {bluer }}\right)$ is comparable with the deflection of the pointer $\bar{X}$, it becomes difficult to distinguish between the spin up and spin down results, which were previously registered. Thus taking $\bar{X}^{2} / \Delta^{2} \gg 1$, which is a necessary condition for a meaningful pointer, and $\xi(t) \simeq 1$, we have

$$
t_{\text {bluer }} \simeq \tau_{r}(\bar{X} / \bar{\sigma})^{2}
$$


This "theoretical" time of bluer is a consequence of the classical Brownian-like diffusion of the pointer, and since always $\bar{X} \gg \bar{\sigma}$, this time is much greater then the relaxation time.

We turn, now, to the off-diagonal term of the spatial probability of the pointer, namely to the interference term, which is a purely quantum mechanical effect, like that of Eq. (28). We set, again, in Eq. (139), $\mathbf{R}^{\prime}=\mathbf{R}$, but now take $\sigma^{\prime}=-1$, and $\sigma=+1$, and have

$$
\begin{aligned}
\Omega^{\prime} & =\mathbf{R}+\widehat{\mathbf{x}} \bar{X}, \\
\mathbf{\Omega} & =\mathbf{R}-\widehat{\mathbf{x}} \bar{X},
\end{aligned}
$$

thus using Eqs. (142) and (144) we get an expression similar to that of Eq. (28), i.e.,

$$
P_{-1,+1}(\mathbf{R}, t)=2 \sqrt{P_{+}(\mathbf{R}, t) P_{-}(\mathbf{R}, t)} \cos \left(\frac{\mathbf{R} \cdot \overline{\mathbf{X}}}{\Delta_{\beta}^{2}(t)} \frac{t}{\tau_{f}}+\varphi_{-}-\varphi_{+}\right) \exp \left\{-\frac{\bar{X}^{2}\left(\beta^{2} / \Delta^{2}\right)}{2 \Delta_{\beta}^{2}(t)}\right\}
$$

Comparing this interference probability with that of the free pointer, Eq. (28), we notice that in Eq. (147), beside the replacement of $\Delta_{f}^{2}(t)$ by $\Delta_{\beta}^{2}(t)$, an additional exponential factor, $e^{-g(t)}$, appears, with

$$
g(t)=\frac{\bar{X}^{2}\left(\beta^{2} / \Delta^{2}\right)}{2 \Delta_{\beta}^{2}(t)}
$$

This damping effect stems, uniquely, from the interaction with the bath, and it is similar to that found in Refs. [6,8]. This exponential factor will kill the interference term almost instantaneously. To show it, we rewrite the damping function, $g(t)$, of Eq. (147) as

$$
g(t)=\frac{1}{2}\left(\frac{\bar{X}}{\Delta}\right)^{2} \frac{\left(\frac{\bar{\sigma}}{\Delta}\right)^{2} \frac{t}{\tau_{r}}}{1+\left(\frac{\bar{\sigma}}{\Delta}\right)^{2} \frac{t}{\tau_{r}}}
$$

where we took $\xi(t)=1$. First we investigate $g(t)$, at early times, when $t \ll \tau_{r}\left(\frac{\Delta}{\bar{\sigma}}\right)^{2}$. We see that $g(t) \rightarrow t / \tau_{\text {int }}$, where the interference damping time is given by

$$
\tau_{\text {int }}=2 \tau_{r}\left(\frac{\Delta}{\bar{X}}\right)^{2}\left(\frac{\Delta}{\bar{\sigma}}\right)^{2}
$$

which is indeed extremely small compared to $\tau_{r}$. To make a simple estimate let us take the relevant parameters for a silver atom pointer i.e. $\Delta \sim 1 \mu \mathrm{m}, \bar{\sigma} \sim 0.1 \mu \mathrm{m}$ and $\bar{X} \sim 1$ 
$\mathrm{cm}$.This yields $\tau_{\text {int }} / \tau_{r} \simeq 10^{-10}$. It is interesting to compare Eq. (149) with Eq. (86) of the gas reservoir model. We see that both expressions are formally identical. Taking $D=\gamma M k_{B} T / \hbar^{2}$ and $\tau_{f}=2 M \Delta^{2} / \hbar$ we can write the term containing $t$ in the nominator of Eq. (86) as

$$
2 D\left(\frac{2 \Delta}{\gamma \tau_{f}}\right)^{2} t=2\left(\frac{1}{\Delta}\right)^{2} \frac{k_{B} T}{M \gamma^{2}} \gamma t .
$$

The analogous term in Eq. (149) gives

$$
\left(\frac{1}{\Delta}\right)^{2} \frac{\bar{\sigma}^{2}}{\tau_{r}} t=2\left(\frac{1}{\Delta}\right)^{2} \frac{k_{B} T}{M \gamma^{2}} \gamma t,
$$

where we have taken $\bar{\sigma}^{2} \rightarrow 2 k_{B} T /\left(M \nu^{2}\right)$, and $\nu=\gamma=1 / \tau_{r}$. We notice that in the regime where $g(t)$ is linear in time both the Fokker - Planck and the random field models give the same behavior. Of course when the time $t$ increases, so does $g(t)$, and eventually it reaches a saturation value of $g(t) \rightarrow \frac{1}{2}(\bar{X} / \Delta)^{2}$. 


\section{REFERENCES}

[1] W. H. Zurek, Phys. Rev. D 26, 1862 (1982).

[2] W. H. Zurek, Physics Today 42, 36 (1991).

[3] W. H. Zurek, Physics World, January, 25 (1997).

[4] H. D. Zeh, Found. Phys. 1, 69 (1970); see also H. D. Zeh, Found. Phys. 3, 109 (1973).

[5] O. Kübler and H. D. Zeh, Ann. Phys. (N.Y.) 76, 405 (1973).

[6] A. O. Caldeira and A. J. Leggett, Phys. Rev. A 31, 1059 (1985).

[7] A. O. Caldeira and A. J. Leggett, Physica A 121, 587 (1983).

[8] D. F. Walls and G. J. Milburn, Phys. Rev. A 31, 2043 (1985).

[9] D. F. Walls, M. J. Collet and G. J. Milburn, Phys. Rev. D 32, 3208 (1985).

[10] W. H. Zurek, S. Habib, and J.P.Paz, Phys. Rev. Lett. 70, 1187 (1993).

[11] M. J. Collet, Phys. Rev. A 38, 2233 (1988).

[12] M. Brune et al., Phys. Rev. Lett. 77, 4887 (1996); see also Phys. Today 51, No. 7, 36 (1998).

[13] J.P.Paz, and W. H. Zurek, Phys. Rev. Lett. 82, 5181 (1999).

[14] A. Venugopalan, Phys. Rev. A 61, 012102, (2000); see also A. Venugopalan, D. Kumar, R. Ghosh, Physica A220, 563 (1995), and A. Venugopalan, Phys. Rev. A 56, 4307 (1997).

[15] A. Peres, Phys. Rev. D 39, 2943 (1989).

[16] C. Cohen-Tannoudji, J. Dupont-Roc, G. Grynberg, in Atom - Photon Interactions, Basic Processes and Applications, (John Wiley and Sons, 1992), pages 262-271.

[17] W. G. Unruh and W. H. Zurek, Phys. Rev. D 40, 1071 (1989). 
[18] E. Joos, Phys. Rev. D 29, 1626 (1984).

[19] S. Chandrasekhar, Rev. Mod. Phys. 15, 1 (1943). 


\section{FIGURE CAPTIONS}

\section{Fig. 1}

A graphical description of the decoherence function, $g(t)$, in the exponent of Eq. (81), normalized to the saturation value $\frac{1}{2}(\bar{X} / \Delta)^{2}$ and as a function of $\gamma t$. In the inset, we see more closely the behavior at initial times, where $g(t)$ starts as $\left(\Gamma^{\prime} t\right)^{3}$ and quickly goes to the linear behavior as $\Gamma t$. 


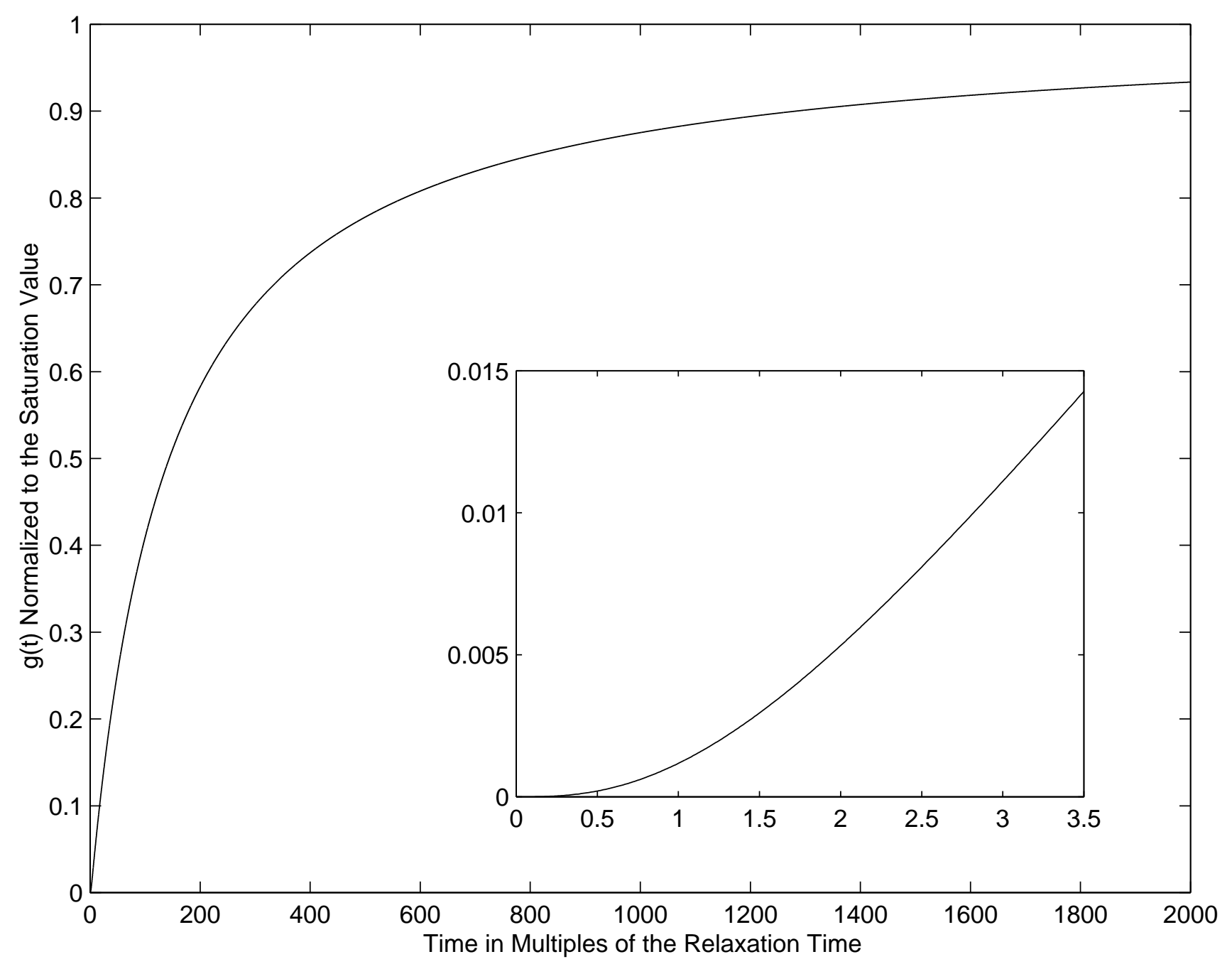

